\title{
Optimal Contracting with Effort and Misvaluation *
}

\author{
Agostino Capponi \\ School of Industrial Engineering \\ Purdue University, West Lafayette, IN 47907 \\ Email: capponi@purdue.edu
}

Jakša Cvitanić

Division of the Humanities and Social Sciences

California Institute of Technology, Pasadena, CA 91125

Email: cvitanic@hss.caltech.edu

Türkay Yolcu

Department of Mathematics

Purdue University, West Lafayette, IN 47907

Email: tyolcu@math.purdue.edu

May 27, 2012

\begin{abstract}
We propose a new continuous time contracting model, where the project value process can only be observed with noise, and there are two sources of moral hazard: effort and misvaluation. We consider an economic scenario where principal and agent can alter the fundamental value of the firm through misvaluation, thus increasing the price estimated by the market. In doing so, the latter uses a level of effort and misvaluation that he believes the agent will apply. We specialize the framework to analyze in detail two cases, namely when the market can only correctly anticipate the effort, and when it can correctly anticipate both. In the first case, we find that it is optimal for the principal to induce the agent to misvalue the firm. Using calculus of variation techniques, we recover the optimal pay-per-performance sensitivity (PPS) of the contract, optimal effort and misvaluation action, as the solution of a second order ordinary differential equation. In the second case, we find that it is not in the interest of the principal and agent to fool the market through misvaluation.
\end{abstract}

Keywords: Optimal contracts; Principal-Agent problem; Variational calculus; Stochastic filtering; Moral hazard.

*The research of J. Cvitanić was supported in part by NSF grants DMS 10-08219. 


\section{Introduction}

We propose a new continuous time contracting model for modeling the principal-agent relationship in which there are two possible actions that the agent can take, namely effort and misvaluation. Following the seminal work of Holmstrom and Milgrom (1987), extended along several directions, Schättler and Sung (1993, 1997), Sung (1995, 1997), Müller (1998), and Cvitanić, Wang and Zhang (2009), we allow the agent to control the drift of the project value process through effort. Differently from the previous works, we allow the agent to fool the market by providing the illusion of growth. For example, the agent can perform accounting manipulations, hide debt, and over hype new investment opportunities, so to con the market into believing that the real price is higher than the fundamental value of the firm. The Enron case serves as a good illustration of this mechanism. At the time of Enron's peak market value of $\$ 70$ billion, the company was actually worth about $\$ 30$ billion. But senior managers attempted to defend the $\$ 40$ billion of excess valuation through off-balance sheet partnerships, and promises to enter into the broadband market in the future. We refer the reader to Jensen (2004) for a more extensive discussion of the drivers of equity overvaluation, along with concrete real world examples, and potential solutions relying on improvement of the corporate governance system.

Concretely, we model the misvaluation induced by the manager through the above described mechanism by a single process, the so called misvaluation process, which is non contractible, and chosen by the manager in such a way to optimize his and shareholders'utility, exploting the created market inefficiency.

Our contracting framework involves three entities: agent (manager), principal (shareholders) and the market (outside investors, excluding shareholders). The shareholders compensate the manager according to a contract that pays at the end of the horizon. Such contract is contingent on the market estimate of the firm value. Our setting also accounts for the fact that manager and shareholders do not observe the outcome process directly. Following evidence that accounting reports are typically contaminated by accounting noise (see also the models of Duffie and Lando (2001) and Capponi and Cvitanić (2009)), we assume that the manager and the shareholders can only observe a white noise contaminated version of the actual output. Moreover, while managerial effort cannot be directly contracted upon by the principal, she can either observe it or compute it correctly in equilibrium.

The proposed framework allows studying moral-hazard problems, where the outside investors are unable to infer the exact level of effort and misvaluation choosen by the agent. This may happen, for instance, due to imperfect knowledge of managerial objectives, such as utility or penalty functions. More specifically we assume that, given its own perceived level of agent's effort and misvaluation, the market produces a price estimate. If the optimal effort and misvaluation action applied by the agent differs from the corresponding assumed market levels, the resulting market price would be a biased estimate of the fundamental value. We assume that the utility functions of the principal and of the agent are exponential. We impose a quadratic cost on the agent, for both the misvaluation and the effort action. The cost for misvaluation captures both the negative consequences on the manager reputation, as well as all the endeavours of the agent in generating the illusion of growth.

We consider the class of linear contracts, and assume deterministic effort and misvaluation 
actions for tractability reasons. 1

We specialize our framework to deal with two study cases in detail, both of which assuming that the effort can be correctly anticipated by the market (as noted in Gibbons (2010), for the case of a CEO, effort corresponds to taking actions that increase the shareholder value, which are typically observable by the market). Next, we describe in detail the two scenarios considered

- The market correctly anticipates the effort level, but ignores misvaluation, thus assuming that market value is in line with the fundamental value. Such a setting is representative of an economic scenario where (1) principal and agent have a short term horizon, and (2) the market is largely composed of unsophisticated investors. This captures real cases, such as the massive overvaluation of equity that occurred in the late 1990s and early 2000s, where society overvalued hightech, telecommunications, and internet ventures. Further, as reported in Jensen (2004), such catastrophic overvaluation was also the result of misleading data from managers, not detected by large numbers of naive investors. This is also in line with findings in Balsam et al. (2002), who show that unsophisticated investors often respond some time after the filing date of the Form 10-Q. Clearly, in a long term, even an unsophisticated market would find out that such high growth was an illusion, at which point the firm's value will fall precipitously because all the overvaluation disappears, effectively creating a bubble. However, we assume that this happens after the assumed horizon, and thus it is not modeled here.

- The market correctly anticipates both dimensions of the moral hazard, effort and misvaluation. Such a setting is representative of an economic scenario where investors in the market are sophisticated enough to discover misvaluation immediately .

We notice that our framework differs from the classical "hidden diversion/hidden action" setup a la De Marzo and Sannikov (2006) or Biais et al. (2007). In their setups, both the principal and the agent observe reported cash flows that are contractible, while the agent observes the true cash flows and could divert a portion of them for his personal consumption. Hence, cash diversion is only in the interest of the agent. Using the revealation principle, they show that the principal can always induce the agent to truthfully reveal, given that he can offer a contract which leaves the agent indifferent between diverting and truthfully reporting, while yielding strictly higher payoff for herself. This captures situations, such as the Parmalat accounting scandal, see Ferrarini and Giudici (2005), where the managerial team diverted substantial amount of company cash for their own benefit. Differently, in our framework, no cash diversion occur. Rather, both principal and agent benefit from misvaluation, the first because he would be able to sell the firm at the end of the time horizon at a premium over the fundamental value, and the second because he would see his compensation increase.

In case when the market does not anticipate misvaluation, we are able to fully characterize the optimal amount applied by the agent, along with the effort level and the pay-perperformance sensitivity of the contract, as the solution of a singular second order differential

\footnotetext{
${ }^{1}$ In the classical Holmstrom-Milgrom model, the optimal effort is, in fact, deterministic and the optimal contract is linear. It is possible that also in our model these assumptions are without loss of generality, but we have not been able to prove it.
} 
equation. We find that the level of effort applied by the agent is always higher than the one he would apply in Holmstrom and Milgrom (1987). Similarly, the agent optimally applies an amount of misvaluation higher than the one applied in the contracting framework proposed in Capponi et al. (2011), where no effort is present. We also find that the agent is compensated by the principal with a higher pay-per-performance sensitivity (PPS) relative to the one received when only one of the two actions may be induced. We find that the dependence of PPS on the accounting noise intensity is not always monotonically decreasing, but dependent on the relation between effort and misvaluation cost. This is because while the benefit of misvaluation decreases with the level of accounting noise, the effort action can still increase the market price. This, in turn, induces the principal to give the agent more incentives to apply effort relative to misvaluation, especially if the former has smaller cost than the latter. If the market is aware of misvaluation, the principal cannot induce the agent to misvalue the fundamentals. Intuitively, this happens because misvaluation is no longer in the interest of the agent. Indeed, the latter would no longer see his compensation increase given that the market price becomes an unbiased version of the fundamental value. Therefore, such an action would only come at a cost without giving the agent any benefit.

The rest of the paper is organized as follows. Section 2 presents the filtering model used by the market to compute the price of the firm. Section 3 introduces the general contracting framework. Section 4 specializes the framework to deal with unsophisticated investors, who ignore accounting manipulations. Section 5 analyzes the case where the market is sophisticated and correctly anticipate both effort and misvaluation. Section 6 performs a numerical study to analyze the dependence of the optimal pay-per-performance sensitivity, effort, and misvaluation on the parameters of our model. Section 7 concludes the paper.

\section{The Filtering Model}

Our model consists of the state process and the observations process 2 Let $\left(W_{t}, Z_{t}^{0}\right)$ be a standard two-dimensional Brownian motion on a probability space $(\Omega, \mathcal{F}, \mathbb{P})$, where $\left(\mathcal{F}_{t}\right)_{0 \leq t \leq T}$ is the filtration generated by it, up to time $T>0$. Let

$$
x_{t}=\epsilon t+\epsilon W_{t}
$$

We assume the "mean variance trade-off" of the output process $\epsilon$ to be constant, and refer to Cvitanić and Zhang (2007) for the case when $\epsilon$ can be controlled. For simplicity, we assume that $x_{0}$ is observed. The state process, or output process, $x_{t}$ is not directly observed by principal and agent, due to the presence of accounting noise, and both observe the process $y$ given by

$$
d y_{t}=x_{t} d t+\sigma d Z_{t}^{0}
$$

where $\sigma>0$ is the intensity of the accounting noise. We next model the misvaluation applied by the agent. Denote by $\mathcal{F}_{t}^{y}$ the filtration generated by the observation process $\left\{y_{t}\right\}$. For given

\footnotetext{
${ }^{2}$ In what follows, subscript $t$ denotes the time variable, not a partial derivative, for which we use $\partial$ operator.
} 
deterministic bounded functions $u_{t}$ and $a_{t}$, let us define

$$
\begin{aligned}
& W_{t}^{u} \triangleq W_{t}-\int_{0}^{t} u_{s} d s, \quad Z_{t}^{a} \triangleq Z_{t}^{0}-\int_{0}^{t} \frac{a_{s}}{\sigma} d s \\
& M_{t}^{u, a} \triangleq \exp \left\{\int_{0}^{t} u_{s} d W_{s}-\frac{1}{2} \int_{0}^{t} u_{s}^{2} d s\right\} \exp \left\{\int_{0}^{t} \frac{a_{s}}{\sigma} d Z_{s}^{0}-\frac{1}{2} \int_{0}^{t}\left(\frac{a_{s}}{\sigma}\right)^{2} d s\right\} \\
& \frac{d \mathbb{P}^{u, a}}{d \mathbb{P}}=M_{T}^{u, a}
\end{aligned}
$$

Then, by Girsanov theorem, $M_{t}^{u, a}$ is a martingale and $\mathbb{P}^{u, a}$ is a probability measure under which $\left(W_{t}^{u}, Z^{a}\right)$ is a $\mathbb{P}^{u, a}$ Brownian motion. Moreover, we have the dynamics

$$
\begin{gathered}
d x_{t}=u_{t} \epsilon d t+\epsilon d W_{t}^{u}, \\
d y_{t}=\left(x_{t}+a_{t}\right) d t+\sigma d Z_{t}^{a}
\end{gathered}
$$

In other words, the agent improves the return of the output by his effort $u_{t}$ and misvaluates by $a_{t}$ the fundamental value $x_{t}$. Although Eq. (2.4) suggests that misvaluation has persistent effects in our model, we remark that persistency is not long-lived, given that the economic scenario we have in mind has a short horizon.

Let us introduce the notation $\mathbb{E}^{u, a}[X] \triangleq \mathbb{E}^{\mathbb{P}^{u, a}}[X]$, where $X$ is a given random variable. Then, introduce the filter process

$$
\hat{x}_{t}^{u, a} \triangleq \mathbb{E}^{u, a}\left[x_{t} \mid \mathcal{F}_{t}^{y}\right]
$$

By standard filtering theory, we have that the conditional expectation is governed by the following SDE

$$
d \hat{x}_{t}^{u, a}=\left(\epsilon u_{t}\right) d t+\frac{v_{t}}{\sigma} d \hat{Z}_{t}^{u, a}
$$

where

$$
\hat{Z}_{t}^{u, a}=Z_{t}^{a}+\frac{1}{\sigma} \int_{0}^{t}\left(x_{s}-\hat{x}_{s}^{u, a}\right) d s .
$$

Remark 2.1. We have that $\hat{x}_{t}^{u, a}$ is the estimate of the output value at time $t$, assuming that the market can perfectly infer the effort $u_{s}$ and the misvaluation $a_{s}, 0 \leq s \leq t$, applied by the agent. If the market cannot perfectly measure the actual effort $u_{t}$ and misvaluation $a_{t}$ applied by the agent, and were to use estimates $\tilde{u}_{t}$ and $\tilde{a}_{t}$ instead, then it would value the firm as $\hat{x}_{t}^{\tilde{u}, \tilde{a}}$.

We next state a useful lemma, which will be later used to compute the optimal contract. The proof is reported in Appendix A. Introduce the notation

$$
v_{t}=\epsilon \sigma \tanh \left(\frac{\epsilon t}{\sigma}\right)
$$

Then, we have 


\section{Lemma 2.1.}

$$
\hat{Z}_{t}^{\tilde{u}, \tilde{a}}=\int_{0}^{t} \frac{\sigma}{v_{s}} d \hat{x}_{s}^{\tilde{u}, \tilde{a}}-\int_{0}^{t} \frac{\epsilon \sigma \tilde{u}_{s}}{v_{s}} d s
$$

and so $\hat{Z}_{t}^{u, a}$ is expressed in terms of $\hat{x}_{t}^{\tilde{u}, \tilde{a}}$ as

$$
\hat{Z}_{t}^{u, a}=\hat{Z}_{t}^{\tilde{u}, \tilde{a}}+\int_{0}^{t} \frac{\tilde{a}_{s}-a_{s}}{\sigma} d s+\int_{0}^{t} \frac{\hat{x}_{s}^{\tilde{u}, \tilde{a}}-\hat{x}_{s}^{u, a}}{\sigma} d s
$$

Moreover, it can be shown, see Appendix $\mathrm{A}$, that

Lemma 2.2. The following relation hold

$$
\hat{x}_{t}^{\tilde{u}, \tilde{a}}-\hat{x}_{t}^{u, a}=\operatorname{sech}\left(\frac{\epsilon t}{\sigma}\right) \int_{0}^{t}\left[\epsilon \cosh \left(\frac{\epsilon s}{\sigma}\right)\left(\tilde{u}_{s}-u_{s}\right)+\frac{\epsilon}{\sigma} \sinh \left(\frac{\epsilon s}{\sigma}\right)\left(a_{s}-\tilde{a}_{s}\right)\right] d s
$$

where, we recall that the hyperbolic secant is defined by $\operatorname{sech}(x)=\frac{1}{\cosh (x)}$.

Using Eq. (2.8) and Eq. (2.10), Eq. (2.9) may be rewritten as

$$
\begin{aligned}
\hat{Z}_{t}^{u, a}= & \int_{0}^{t} \frac{\sigma}{v_{s}} d \hat{x}_{s}^{\tilde{u}, \tilde{a}}-\int_{0}^{t} \frac{\epsilon \sigma u_{s}}{v_{s}} d s+\int_{0}^{t} \frac{\tilde{a}_{s}-a_{s}}{\sigma} d s+ \\
& \frac{\epsilon}{\sigma^{2}}\left(\int_{0}^{t}\left[\operatorname{sech}\left(\frac{\epsilon s}{\sigma}\right)\left(\int_{0}^{s} \sinh \left(\frac{\epsilon r}{\sigma}\right)\left(a_{r}-\tilde{a}_{r}\right) d r\right)\right] d s\right)+ \\
& \frac{\epsilon}{\sigma} \int_{0}^{t}\left[\operatorname{sech}\left(\frac{\epsilon s}{\sigma}\right) \int_{0}^{s} \cosh \left(\frac{\epsilon r}{\sigma}\right)\left(\tilde{u}_{r}-u_{r}\right) d r\right] d s
\end{aligned}
$$

Moreover, we have that

$$
\begin{aligned}
d \hat{x}_{t}^{\tilde{u}, \tilde{a}=} & \epsilon \tilde{u}_{t} d t+\frac{v_{t}}{\sigma} d \hat{Z}_{t}^{u, a}+\frac{v_{t}}{\sigma^{2}}\left(a_{t}-\tilde{a}_{t}\right) d t-\frac{v_{t}}{\sigma^{2}}\left(\hat{x}_{t}^{\tilde{u}, \tilde{a}}-x_{t}^{u, a}\right) d t \\
= & \epsilon \tilde{u}_{t} d t+\frac{v_{t}}{\sigma} d \hat{Z}_{t}^{u, a}+\frac{v_{t}}{\sigma^{2}}\left(a_{t}-\tilde{a}_{t}\right) d t-\frac{v_{t}}{\sigma^{2}}\left(\frac{\epsilon}{\sigma} \operatorname{sech}\left(\frac{\epsilon t}{\sigma}\right) \int_{0}^{t} \sinh \left(\frac{\epsilon r}{\sigma}\right)\left(a_{r}-\tilde{a}_{r}\right) d r+\right. \\
& \left.\epsilon \operatorname{sech}\left(\frac{\epsilon t}{\sigma}\right) \int_{0}^{t} \cosh \left(\frac{\epsilon r}{\sigma}\right)\left(\tilde{u}_{r}-u_{r}\right) d r\right)
\end{aligned}
$$

where the first equality follows plugging (2.9) inside the filter estimate given in (2.5), while the second equality follows using relation (2.10).

\section{The Contracting Model}

We consider a principal agent model in which the principal can induce the agent to (1) increase the fundamental value of the firm by applying more and effort, and (2) alter the fundamental value through misvaluation, thus disaligning the market price and fundamental value. We consider linear contracts of the form

$$
\mathscr{C}_{T}=c+\int_{0}^{T} \alpha_{t} d \hat{x}_{t}^{\tilde{u}, \tilde{a}}
$$


with $\alpha$ being deterministic. Here $x_{t}^{\tilde{u}, \tilde{a}}$ is the project value estimate computed by the market using its own perceived level of effort $\tilde{u}$, and misvaluation $\tilde{a}$, which may differ from the optimal levels applied by the agent.

We note that in Holmstrom and Milgrom (1987), the optimal contract is linear in the true final output value. In our case the latter has to be estimated using the whole history of observations. It is then not surprising that the optimal linear contract will be a functional of the whole observation history, and not just of the terminal value. The contract payment $\mathscr{C}_{T}$ is an $\mathcal{F}_{T}^{y}$-measurable random variable, and should be interpreted as a payment in cash, the amount of which depends on the random outcomes of $\hat{x}^{\tilde{u}, \tilde{a}}$ up to time $T$. In what follows, we impose the following restriction on the admissible actions.

Assumption 3.1. The set of admissible effort action $u_{t}$, misvaluation action $a_{t}$ and payper-performance sensitivity $\alpha_{t}$ is $C^{1}[0, T]$, the set of deterministic continuously differentiable functions on the interval $[0, T]$.

For latter use, denote

$$
m_{T} \triangleq \max _{t \in[0, T]}\left\{\left|a_{t}\right|,\left|u_{t}\right|,\left|\alpha_{t}\right|\right\}<+\infty
$$

We assume that the agent incurs a quadratic cost for both misvaluation and effort action. More specifically, we denote by $\mathscr{E}_{T}$ the cost incurred for his effort action in interval $[0, T]$, given by

$$
\mathscr{E}_{T}=\frac{k}{2} \int_{0}^{T} u_{t}^{2} d t
$$

where $k$ is a positive constant. We denote by $\mathscr{G}_{T}$ the penalty incurred for his misvaluation action in interval $[0, T]$, given by

$$
\mathscr{G}_{T}=\frac{\xi}{2} \int_{0}^{T} a_{t}^{2} d t
$$

where $\xi$ is a positive constant.

Remark 3.2. Broadly speaking, the cost of undervaluation is typically lower than that of overvaluation, and thus a non-symmetric cost function should be used. However, in our model, it appears that principal and agent always find it optimal to induce the market to overvalue the firm, as it can be seen from the various graphs reporting the behavior of the optimal $a_{t}$ in Section [6. Therefore, following the celebrated Holmstrom and Milgrom (1987), who adopts a symmetric quadratic cost for the (positive) effort, we also employ a quadratic cost for misvaluation.

We consider exponential utilities

$$
U_{1}(x)=-e^{-\gamma_{1} x} \quad U_{2}(x)=-e^{-\gamma_{2} x}
$$

Before continuing, we remark that the Lagrangians considered in this paper belong to a special class, called Tonelli Lagrangians, whose definition is given, for instance, in Clarke (1989). Theorem B.2, given in Appendix B, and rephrased from Tonelli's existence theorem, Corollary 2.5 and Proposition 2.1 in Clarke (1989), summarizes existence, uniqueness and regularity of the minimizer $\mathbf{x}_{t}^{*}$ of $\min _{\mathbf{x}_{t}} \int_{0}^{T} L\left(t, \mathbf{x}_{t}, \mathbf{x}_{t}^{\prime}\right) d t$. 


\subsection{The agent optimization problem}

The agent minimizes over $u_{t}$ and $a_{t}$ the following quantity

$$
\begin{aligned}
& \mathbb{E}^{u, a}\left[U_{1}\left(\mathscr{C}_{T}-\mathscr{G}_{T}-\mathscr{E}_{T}\right)\right]= \\
& \mathbb{E}^{u, a}\left[-\exp \left\{-\gamma_{1}\left(c+\int_{0}^{T} \alpha_{t} d \hat{x}_{t}^{\tilde{u}, \tilde{a}}-\frac{1}{2} \int_{0}^{T}\left(\xi a_{t}^{2}+k u_{t}^{2}\right) d t\right)\right\}=\right. \\
& -\exp \left\{-\gamma_{1}\left(c+\int_{0}^{T} \alpha_{t}\left(\epsilon \tilde{u}_{t}-\frac{1}{2} \alpha_{t} \gamma_{1} \frac{v_{t}^{2}}{\sigma^{2}}\right) d t\right)\right\} \\
& \exp \left\{-\gamma_{1} \int_{0}^{T} \alpha_{t} \frac{v_{t}}{\sigma^{2}}\left(\left(a_{t}-\tilde{a}_{t}\right)-\left(\frac{\epsilon}{\sigma} \operatorname{sech}\left(\frac{\epsilon t}{\sigma}\right) \int_{0}^{t} \sinh \left(\frac{\epsilon r}{\sigma}\right)\left(a_{r}-\tilde{a}_{r}\right) d r\right)\right) d t\right\} \\
& \exp \left\{-\gamma_{1} \int_{0}^{T} \alpha_{t} \frac{\epsilon v_{t}}{\sigma^{2}} \operatorname{sech}\left(\frac{\epsilon t}{\sigma}\right) \int_{0}^{t} \cosh \left(\frac{\epsilon r}{\sigma}\right)\left(\tilde{u}_{r}-u_{r}\right) d r\right\} \exp \left\{\frac{\gamma_{1}}{2} \int_{0}^{T}\left(\xi a_{t}^{2}+k u_{t}^{2}\right) d t\right\}
\end{aligned}
$$

where the last equality follows by using (2.12) and the fact that, for a given deterministic function $\psi(t)$, we have

$$
\mathbb{E}^{u, a}\left[\exp \left(\int_{0}^{T} \psi(t) d \hat{Z}_{t}^{u, a}\right)\right]=\exp \left(\frac{1}{2} \int_{0}^{T} \psi(t)^{2} d t\right)
$$

Equivalently, we want to maximize over $u_{t}$ and $a_{t}$ the functional given by

$$
\int_{0}^{T} \mathcal{L}\left(t, u_{t}, a_{t}\right) d t
$$

where

$$
\begin{aligned}
\mathcal{L}\left(t, u_{t}, a_{t}\right)= & \alpha_{t}\left(\tilde{u}_{t} \epsilon-\frac{\gamma_{1}}{2} \alpha_{t} \frac{v_{t}^{2}}{\sigma^{2}}+\frac{v_{t}\left(a_{t}-\tilde{a}_{t}\right)}{\sigma^{2}}-\frac{\epsilon v_{t}}{\sigma^{3}} \operatorname{sech}\left(\frac{\epsilon t}{\sigma}\right) \int_{0}^{t} \sinh \left(\frac{\epsilon s}{\sigma}\right)\left(a_{s}-\tilde{a}_{s}\right) d s\right. \\
& \left.+\frac{\epsilon v_{t}}{\sigma^{2}} \operatorname{sech}\left(\frac{\epsilon t}{\sigma}\right) \int_{0}^{t} \cosh \left(\frac{\epsilon s}{\sigma}\right)\left(\tilde{u}_{s}-u_{s}\right) d s\right)-\left(\frac{k}{2} u_{t}^{2}+\frac{\xi}{2} a_{t}^{2}\right)
\end{aligned}
$$

and $v_{t}$ is defined by Eq. (2.7).

\subsection{The principal optimization problem}

The optimization problem of the principal is to maximize over $\alpha_{t} \in C^{1}[0, T]$ her utility under the reservation constraint that the agent expected utility given by Eq. (3.4) is equal to $R_{0}<0$. From this constraint, using Eq. (2.12) and (3.4), we obtain

$$
\begin{aligned}
-R_{0} & =e^{-\gamma_{1} c} \mathbb{E}^{u, a}\left[-e^{-\gamma_{1} \int_{0}^{T} \alpha_{t} d \hat{x}_{t}^{\tilde{u}, \tilde{a}}+\frac{1}{2} \gamma_{1} \int_{0}^{T}\left(k u_{t}^{2}+\xi a_{t}^{2}\right) d t}\right] \\
& \left.\left.\left.=e^{-\gamma_{1} c} e^{-\gamma_{1}\left[\int _ { 0 } ^ { T } \alpha _ { t } \left(\epsilon \tilde{u}_{t}-\frac{1}{2} \alpha_{t} \gamma_{1} \frac{v_{t}^{2}}{\sigma^{2}}+\frac{v_{t}\left(a_{t}-\tilde{a}_{t}\right)}{\sigma^{2}}-\frac{v_{t}}{\sigma^{2}}\left(\hat{x}_{t}^{\tilde{u}}, \tilde{a}\right.\right.\right.}-\hat{x}_{t}^{u, a}\right)\right) d t-\frac{1}{2} \int_{0}^{T}\left(k u_{t}^{2}+\xi a_{t}^{2}\right) d t\right]
\end{aligned}
$$


leading to

$$
e^{-\gamma_{1} c}=-R_{0} e^{\gamma_{1}\left[\int_{0}^{T} \alpha_{t}\left(\epsilon \tilde{u}_{t}-\frac{1}{2} \alpha_{t} \gamma_{1} \frac{v_{t}^{2}}{\sigma^{2}}+\frac{v_{t}\left(a_{t}-\tilde{a}_{t}\right)}{\sigma^{2}}-\frac{v_{t}}{\sigma^{2}}\left(\hat{x}_{t}^{\tilde{u}, \tilde{a}}-\hat{x}_{t}^{u, a}\right)\right) d t-\frac{1}{2} \int_{0}^{T}\left(k u_{t}^{2}+\xi a_{t}^{2}\right) d t\right]}
$$

and therefore, solving for $c$ in the above equation, we obtain

$c=-\frac{1}{\gamma_{1}} \log \left(-R_{0}\right)-\int_{0}^{T}\left(\alpha_{t}\left[\epsilon \tilde{u}_{t}-\frac{1}{2} \frac{v_{t}^{2}}{\sigma^{2}} \gamma_{1} \alpha_{t}+\frac{v_{t}\left(a_{t}-\tilde{a}_{t}\right)}{\sigma^{2}}-\frac{v_{t}}{\sigma^{2}}\left(\hat{x}_{t}^{\tilde{u}, \tilde{a}}-\hat{x}_{t}^{u, a}\right)\right]-\frac{1}{2} k u_{t}^{2}-\frac{1}{2} \xi a_{t}^{2}\right) d t$

Plugging it into the principal's utility, and using again Eq. (2.12) and (3.4), we obtain that the principal maximizes over $\alpha_{t}$ the following quantity

$$
\begin{aligned}
U_{P}\left(\hat{x}_{T}^{\tilde{u}, \tilde{a}}-\mathscr{C}_{T}\right)= & \mathbb{E}^{u, a}\left[-\exp \left\{-\gamma_{2}\left(\hat{x}_{T}^{\tilde{u}, \tilde{a}}-c-\int_{0}^{T} \alpha_{t} d \hat{x}_{t}^{\tilde{u}, \tilde{a}}\right)\right\}\right] \\
= & -\exp \left\{-\gamma_{2} \int_{0}^{T}\left(1-\alpha_{t}\right)\left[\epsilon \tilde{u}_{t}-\frac{1}{2} \gamma_{2}\left(1-\alpha_{t}\right) \frac{v_{t}^{2}}{\sigma^{2}}+\frac{v_{t}\left(a_{t}-\tilde{a}_{t}\right)}{\sigma^{2}}-\frac{v_{t}}{\sigma^{2}}\left(\hat{x}_{t}^{\tilde{u}, \tilde{a}}-\hat{x}_{t}^{u, a}\right)\right] d t\right\} \\
& \exp \left\{-\gamma_{2} \int_{0}^{T} \alpha_{t}\left[\epsilon \tilde{u}_{t}-\frac{1}{2} \alpha_{t} \gamma_{1} \frac{v_{t}^{2}}{\sigma^{2}}+\frac{v_{t}\left(a_{t}-\tilde{a}_{t}\right)}{\sigma^{2}}-\frac{v_{t}}{\sigma^{2}}\left(\hat{x}_{t}^{\tilde{u}, \tilde{a}}-\hat{x}_{t}^{u, a}\right)\right] d t\right\} \\
& \exp \left\{-\frac{\gamma_{2}}{\gamma_{1}} \log \left(-R_{0}\right)\right\} \exp \left\{\frac{1}{2} \gamma_{2}\left(k u_{t}^{2}+\xi a_{t}^{2}\right) d t\right\}
\end{aligned}
$$

where we have used that $\hat{x}_{T}^{\tilde{u}, \tilde{a}}=\int_{0}^{T} d \hat{x}_{t}^{\tilde{u}, \tilde{a}}$. Therefore, the principal wants to maximize the following quantity over $\alpha_{t}$ :

$$
\begin{aligned}
\mathcal{A}\left(t, \alpha_{t}\right)= & \int_{0}^{T}\left[\epsilon \tilde{u}_{t}+\frac{v_{t}}{\sigma^{2}}\left(a_{t}-\tilde{a}_{t}\right)-\frac{v_{t}}{\sigma^{2}}\left(\hat{x}_{t}^{\tilde{u}, \tilde{a}}-\hat{x}_{t}^{u, a}\right)-\frac{v_{t}^{2}}{2 \sigma^{2}}\left(\gamma_{2}\left(1-\alpha_{t}\right)^{2}+\gamma_{1} \alpha_{t}^{2}\right)\right] d t \\
& -\frac{1}{2} \int_{0}^{T}\left[\left(k u_{t}^{2}+\xi a_{t}^{2}\right)\right] d t
\end{aligned}
$$

and $a_{t}$ and $u_{t}$ are, respectively, the optimal misvaluation and effort actions applied by the agent.

\section{The contracting model when market only anticipates effort}

We consider the case when the market correctly anticipates the optimal effort level applied by the agent, but ignores misvaluation. This means that $\tilde{u}_{t}=u_{t}$, and $\tilde{a}_{t}=0$. In particular, the market values the firm using $\hat{x}^{u, 0}$. Under these specifications, we study in detail the optimization problems of the principal and of the agent. We use $u_{t}^{M E}, a_{t}^{M E}$, and $\alpha_{t}^{M E}$ to denote, respectively, the optimal effort, misvaluation, and pay-per-performance sensitivity, where $M E$ indicates the presence of both misvaluation and effort. 


\subsection{The agent}

We characterize the optimal level of effort and misvaluation applied by the agent, when a given contract $C_{T}$ is offered to her. First, we introduce the following function

$$
p_{t}=\int_{0}^{t} \sinh \left(\frac{\epsilon s}{\sigma}\right) a_{s} d s
$$

which implies

$$
p_{0}=0 \quad \text { and } \quad p_{t}^{\prime}=\sinh \left(\frac{\epsilon t}{\sigma}\right) a_{t}
$$

Observe that $p_{t}$ and $p_{t}^{\prime}$ are both bounded by a multiple of $m_{T}$ :

$$
\begin{array}{r}
\max _{t \in[0, T]}\left\{\left|p_{t}\right|\right\} \leq \max _{t \in[0, T]}\left\{\left|a_{t}\right|\right\}\left(\frac{\sigma}{\epsilon} \cosh \left(\frac{\epsilon T}{\sigma}\right)-\frac{\sigma}{\epsilon}\right)<m_{T}\left(\frac{\sigma}{\epsilon} \cosh \left(\frac{\epsilon T}{\sigma}\right)\right)<+\infty \\
\max _{t \in[0, T]}\left\{\left|p_{t}^{\prime}\right|\right\} \leq \max _{t \in[0, T]}\left\{\left|a_{t}\right|\right\} \max _{t \in[0, T]}\left\{\sinh \left(\frac{\epsilon t}{\sigma}\right)\right\}<\left(m_{T}+1\right) \sinh \left(\frac{\epsilon T}{\sigma}\right)<+\infty
\end{array}
$$

Next, we state the main result of this section, which provides an analytic characterization of the optimal effort and misvaluation applied by the agent.

Proposition 4.1. Given a contract $\mathscr{C}_{T}$ offered by the principal to the agent, the optimal misvaluation action $a_{t}^{M E}$ and effort $u_{t}^{M E}$ applied by the agent are given by

$$
\begin{aligned}
a_{t}^{M E} & =\frac{\epsilon}{\sigma \xi} \tanh \left(\frac{\epsilon t}{\sigma}\right) \alpha_{t}-\frac{\epsilon^{2}}{\sigma^{2} \xi} \sinh \left(\frac{\epsilon t}{\sigma}\right) \int_{t}^{T} \tanh \left(\frac{\epsilon s}{\sigma}\right) \operatorname{sech}\left(\frac{\epsilon s}{\sigma}\right) \alpha_{s} d s \\
u_{t}^{M E} & =\frac{\epsilon}{k} \alpha_{t}
\end{aligned}
$$

The proof of Proposition 4.1 is reported in Appendix C.

Remark 4.3. From Eqs. (4.5) and (4.6) we conclude that the optimal effort action $u_{t}^{M E}$ and the optimal misvaluation action $a_{t}^{M E}$ belong to $C^{1}[0, T]$, as long as $\alpha$ is $C^{1}[0, T]$ as in Assumption 3.1.

\subsection{The principal}

When $\tilde{u}_{t}=u_{t}$, and $\tilde{a}_{t}=0$, we obtain that the functional optimizated by the principal, given by Eq. (3.8), reduces to

$$
\begin{aligned}
\mathcal{A}\left(t, \alpha_{t}\right)= & \int_{0}^{T}\left[\epsilon u_{t}^{M E}+\frac{v_{t}}{\sigma^{2}} a_{t}^{M E}-\frac{v_{t}}{\sigma^{2}}\left(\hat{x}_{t}^{u^{M E}, 0}-\hat{x}_{t}^{u^{M E}, a^{M E}}\right)-\frac{v_{t}^{2}}{2 \sigma^{2}}\left(\gamma_{2}\left(1-\alpha_{t}\right)^{2}+\gamma_{1} \alpha_{t}^{2}\right)\right] d t \\
& -\frac{1}{2} \int_{0}^{T}\left[\left(k\left(u_{t}^{M E}\right)^{2}+\xi\left(a_{t}^{M E}\right)^{2}\right)\right] d t
\end{aligned}
$$

where $a_{t}^{M E}$ and $u_{t}^{M E}$ are the optimal misvaluation action and effort given, respectively, by Eq. (4.5) and Eq. (4.6). Using Eq. (4.5), we can rewrite the term $\hat{x}_{t}^{u^{M E}, 0}-\hat{x}_{t}^{u^{M E}, a^{M E}}$ in 
Eq. (2.10) as

$$
\hat{x}_{t}^{u^{M E}, 0}-\hat{x}_{t}^{u^{M E}, a^{M E}}=\frac{\frac{\epsilon^{2}}{\sigma^{2} \xi} \int_{0}^{t}\left[\sinh \left(\frac{\epsilon r}{\sigma}\right) \tanh \left(\frac{\epsilon r}{\sigma}\right) \alpha_{r}-\frac{\epsilon}{\sigma} \sinh ^{2}\left(\frac{\epsilon r}{\sigma}\right)\left(\int_{r}^{T} \tanh \left(\frac{\epsilon s}{\sigma}\right) \operatorname{sech}\left(\frac{\epsilon s}{\sigma}\right) \alpha_{s} d s\right)\right] d r}{\cosh \left(\frac{\epsilon t}{\sigma}\right)}
$$

We next introduce the following functions

$$
\begin{aligned}
\beta_{t} & \triangleq \int_{0}^{t} \sinh \left(\frac{\epsilon s}{\sigma}\right) \tanh \left(\frac{\epsilon s}{\sigma}\right) \alpha_{s} d s \\
\eta_{t} & \triangleq \int_{t}^{T} \tanh \left(\frac{\epsilon s}{\sigma}\right) \operatorname{sech}\left(\frac{\epsilon s}{\sigma}\right) \alpha_{s} d s \\
\zeta_{t} & \triangleq \int_{0}^{t} \sinh ^{2}\left(\frac{\epsilon s}{\sigma}\right) \eta_{s} d s
\end{aligned}
$$

so that

$$
\hat{x}_{t}^{u^{M E}, 0}-\hat{x}_{t}^{u^{M E}, a^{M E}}=\frac{\epsilon^{2}}{\sigma^{2} \xi} \operatorname{sech}\left(\frac{\epsilon t}{\sigma}\right)\left(\beta_{t}-\frac{\epsilon}{\sigma} \zeta_{t}\right) .
$$

In the sequel, we construct the relaxed maximization problem, and regard $\beta, \eta$, and $\zeta$ as three independent variables by adding two Lagrange multipliers through the constraints. Such constraints are obtained using the fundamental theorem of calculus. From Eq. (4.9) and (4.10), we have

$$
\beta_{t}^{\prime}=-\sinh \left(\frac{\epsilon t}{\sigma}\right) \cosh \left(\frac{\epsilon t}{\sigma}\right) \eta_{t}^{\prime}
$$

and from Eq. (4.11) that

$$
\zeta_{t}^{\prime}=\sinh ^{2}\left(\frac{\epsilon t}{\sigma}\right) \eta_{t}
$$

Moreover, we have that $\beta_{T}, \eta_{0}$, and $\zeta_{T}$ are free of constraints, while $\beta_{0}=\eta_{T}=\zeta_{0}=0$. Eq. (4.9), (4.10) and (4.11) turn the integral of Eq. (4.7) into

$$
\mathcal{A}^{P}\left(t, \beta_{t}, \eta_{t}, \zeta_{t}\right)=\int_{0}^{T} x\left(t, \beta_{t}, \eta_{t}, \zeta_{t}, \beta_{t}^{\prime}, \eta_{t}^{\prime}, \zeta_{t}^{\prime}\right) d t
$$


where

$$
\begin{aligned}
X\left(t, \beta_{t}, \eta_{t}, \zeta_{t}, \beta_{t}^{\prime}, \eta_{t}^{\prime}, \zeta_{t}^{\prime}\right)= & \frac{\epsilon^{2}}{k} \operatorname{csch}\left(\frac{\epsilon t}{\sigma}\right) \operatorname{coth}\left(\frac{\epsilon t}{\sigma}\right) \beta_{t}^{\prime} \\
& +\frac{v_{t}}{\sigma^{2}}\left(\frac{\epsilon}{\sigma \xi} \operatorname{csch}\left(\frac{\epsilon t}{\sigma}\right) \beta_{t}^{\prime}-\frac{\epsilon^{2}}{\sigma^{2} \xi} \sinh \left(\frac{\epsilon t}{\sigma}\right) \eta_{t}\right) \\
& -\frac{v_{t}}{\sigma^{3}} \operatorname{sech}\left(\frac{\epsilon t}{\sigma}\right)\left(\frac{\epsilon^{2}}{\sigma \xi} \beta_{t}-\frac{\epsilon^{3}}{\sigma^{2} \xi} \zeta_{t}\right) \\
& -\frac{v_{t}^{2}}{2 \sigma^{2}} \gamma_{1} \operatorname{csch}^{2}\left(\frac{\epsilon t}{\sigma}\right) \operatorname{coth}^{2}\left(\frac{\epsilon t}{\sigma}\right)\left(\beta_{t}^{\prime}\right)^{2} \\
& -\frac{v_{t}^{2}}{2 \sigma^{2}} \gamma_{2}\left(\operatorname{csch}\left(\frac{\epsilon t}{\sigma}\right) \operatorname{coth}\left(\frac{\epsilon t}{\sigma}\right) \beta_{t}^{\prime}-1\right)^{2} \\
& -\frac{\epsilon^{2}}{2 k} \operatorname{csch}^{2}\left(\frac{\epsilon t}{\sigma}\right) \operatorname{coth}\left(\frac{\epsilon t}{\sigma}\right)\left(\beta_{t}^{\prime}\right)^{2} \\
& -\frac{\xi}{2}\left(\frac{\epsilon}{\sigma \xi} \operatorname{csch}\left(\frac{\epsilon t}{\sigma}\right) \beta_{t}^{\prime}-\frac{\epsilon^{2}}{\sigma^{2} \xi} \sinh \left(\frac{\epsilon t}{\sigma}\right) \eta_{t}\right)^{2}
\end{aligned}
$$

Remark 4.4. The maximization problem

$$
\max _{\left(\beta_{t}, \eta_{t}, \zeta_{t}\right)}\left\{\mathcal{A}^{P}\left(t, \beta_{t}, \eta_{t}, \zeta_{t}\right): \beta_{t} \text { and } \eta_{t} \text { satisfy (4.13), } \eta_{t} \text { and } \zeta_{t} \text { satisfy (4.14) }\right\}
$$

is well posed.

The verification of this remark involves tedious computations, and is done in Appendix D. The following theorem is the main result of this section and fully characterizes the quantities needed to compute the optimal PPS and hidden actions.

Theorem 4.1. The maximizers $\beta_{t}, \eta_{t}$ and $\zeta_{t}$ of $\mathcal{A}^{P}$ satisfy

$$
A(t) \eta_{t}^{\prime \prime}+B(t) \eta_{t}^{\prime}+C(t) \eta_{t}+D(t)=0, \quad \eta_{T}=0, \quad \eta_{0}^{\prime}=0
$$

where

$$
\begin{gathered}
A(t)=\epsilon^{2}\left(\gamma_{1}+\gamma_{2}+\frac{1}{\sigma^{2} \xi}\right) \cosh ^{2}\left(\frac{\epsilon t}{\sigma}\right)+\frac{\epsilon^{2}}{k} \operatorname{coth}^{2}\left(\frac{\epsilon t}{\sigma}\right) \cosh ^{2}\left(\frac{\epsilon t}{\sigma}\right) \\
B(t)=\frac{2 \epsilon^{3}}{\sigma}\left(\gamma_{1}+\gamma_{2}+\frac{1}{\sigma^{2} \xi}\right) \sinh \left(\frac{\epsilon t}{\sigma}\right) \cosh \left(\frac{\epsilon t}{\sigma}\right)+\frac{2 \epsilon^{3}}{\sigma k} \operatorname{coth}\left(\frac{\epsilon t}{\sigma}\right)\left(\cosh ^{2}\left(\frac{\epsilon t}{\sigma}\right)-\operatorname{coth}^{2}\left(\frac{\epsilon t}{\sigma}\right)\right) \\
C(t)=\frac{\epsilon^{4}}{\sigma^{4} \xi} \cosh ^{2}\left(\frac{\epsilon t}{\sigma}\right) \\
D(t)=\frac{\epsilon^{3}}{\sigma^{3} \xi} \cosh ^{2}\left(\frac{\epsilon t}{\sigma}\right) \operatorname{sech}\left(\frac{\epsilon T}{\sigma}\right)+\frac{\epsilon^{3}}{\sigma} \cosh \left(\frac{\epsilon t}{\sigma}\right)\left(\gamma_{2}+\frac{1}{k}\left(1-\operatorname{csch}^{2}\left(\frac{\epsilon t}{\sigma}\right)\right)\right)
\end{gathered}
$$

Moreover, we infer that the optimal pay-per performance sensitivity satisfies the initial condition $\alpha_{0}=1$. 
The proof of Theorem 4.1 is reported in Appendix D.

Remark 4.5. Finding the explicit solution of the singular ODE in Eq. (4.17) with the prescribed boundary conditions is not an easy task and accordingly we prefer to analyze the solution of (4.17) numerically. We do so by using SBVP 1.0, a Matlab solver, which provides a collocation solution, based on piecewise polynomial functions, to the singular boundary value problem. The reader is referred to Auzinger et al. (2011) for additional documentation.

Remark 4.6. Due to Eq. (D.23) the maximizer $\alpha_{t}^{M E}$ of $\mathcal{A}\left(t, \alpha_{t}\right)$ is uniquely obtained from

$$
\alpha_{0}^{M E}=1, \quad \alpha_{t}^{M E}=-\operatorname{coth}\left(\frac{\epsilon t}{\sigma}\right) \cosh \left(\frac{\epsilon t}{\sigma}\right) \eta_{t}^{\prime} \quad \text { for } 0<t \leq T
$$

and using Eq. (4.5) and Eq. (4.6) the optimal $a_{t}^{M E}$ and $u_{t}^{M E}$ are uniquely expressed as

$$
\begin{gathered}
a_{0}^{M E}=0, \quad a_{t}^{M E}=-\frac{\epsilon}{\sigma \xi} \cosh \left(\frac{\epsilon t}{\sigma}\right) \eta_{t}^{\prime}-\frac{\epsilon^{2}}{\sigma^{2} \xi} \sinh \left(\frac{\epsilon t}{\sigma}\right) \eta_{t} \quad \text { for } 0<t \leq T \\
u_{0}^{M E}=\frac{\epsilon}{k}, \quad u_{t}^{M E}=-\frac{\epsilon}{k} \operatorname{coth}\left(\frac{\epsilon t}{\sigma}\right) \cosh \left(\frac{\epsilon t}{\sigma}\right) \eta_{t}^{\prime} \quad \text { for } 0<t \leq T
\end{gathered}
$$

Therefore, by Remarks 4.3 and 4.4, we conclude that $\alpha_{t}^{M E}, a_{t}^{M E}$ and $u_{t}^{M E}$ are all at least $C^{1}[0, T]$ functions. Notice that initially, the principal gives full incentive to the agent, who will apply the maximum level of effort. There will be no misvaluation, because we are assuming that $x_{0}$ is common knowledge, and therefore there will be no utility coming from misvaluation.

Not surprisingly, the optimal misvaluation action is nonzero. Our numerical analysis reported in Section 66 shows that it is indeed positive, i.e. principal and agent always induce the market to overvalue the firm. Although our statements have been proven for linear contracts, we conjecture that the qualitative conclusions would remain the same for any optimal contract. Intuitively, this would be the case given that both principal and agent are able to extract positive utility from misvaluation, when the market values the firm without taking it into account.

\subsection{Limiting Cases}

We show that such a contracting framework recovers the well known Holmstrom and Milgrom (1987) model, and the contracting model where only misvaluation is allowed, as special cases. We first recall the expressions for the pay-per-performance sensitivity $\alpha_{t}^{H M}$ and effort $u_{t}^{H M}$ in the Holmstrom-Milgrom model, given by

$$
\alpha_{t}^{H M}=\frac{\gamma_{2}+(1 / k)}{\gamma_{1}+\gamma_{2}+(1 / k)}, \quad u_{t}^{H M}=\frac{\epsilon}{k} \alpha_{t}^{H M}
$$


and the expressions for the pay-per-performance sensitivity $\alpha_{t}^{M}$ and effort $u_{t}^{M}$ in the model with only misvaluation derived in Capponi et al. (2011), given by

$$
\begin{aligned}
\alpha_{t}^{M} & =\frac{\gamma_{2}}{\gamma_{1}+\gamma_{2}}+\frac{\gamma_{1}}{\gamma_{1}+\gamma_{2}} \frac{\operatorname{sech}(\omega T)}{\epsilon \tanh \left(\frac{\epsilon t}{\sigma}\right)}\left[-\omega \sigma \sinh (\omega t)+\epsilon \cosh (\omega t) \tanh \left(\frac{\epsilon t}{\sigma}\right)\right] \\
a_{t}^{M} & =-\frac{\gamma_{1} \omega}{\left(\gamma_{1}+\gamma_{2}\right) \xi} \operatorname{sech}(\omega T) \sinh (\omega t)+\frac{\epsilon}{\sigma \xi} \operatorname{sech}\left(\frac{\epsilon T}{\sigma}\right) \sinh \left(\frac{\epsilon t}{\sigma}\right)
\end{aligned}
$$

where

$$
\omega=\left(\frac{\epsilon^{2} \xi\left(\gamma_{1}+\gamma_{2}\right)}{\left(\gamma_{1}+\gamma_{2}\right) \sigma^{2} \xi+1}\right)^{1 / 2}
$$

In case when there is no observation noise, $\sigma \rightarrow 0$, and the cost of misvaluation, $\xi \rightarrow \infty$, then the actual output is observed, and the agent is discouraged from any misvaluation due to that it becomes very costly. Then, as one would expect, our contracting framework reduces to Holmstrom and Milgrom (1987), as stated in the next proposition.

Proposition 4.2. The following limits hold:

$$
\lim _{\sigma \rightarrow 0}\left(\lim _{\xi \rightarrow \infty} \alpha_{t}^{M E}\right)=\alpha_{t}^{H M}, \quad \lim _{\sigma \rightarrow 0}\left(\lim _{\xi \rightarrow \infty} u_{t}^{M E}\right)=u_{t}^{H M}
$$

The proof of Proposition 4.2 is reported in Appendix E. In the case in which the cost for effort becomes infinite, the principal knows the agent will not apply effort, and therefore only provides incentives for misvaluation. Therefore, we expect the optimal $\alpha_{t}$ to be the same as in the model with only misvaluation, developed in Capponi et al. (2011). This is indeed the case, and we have

Proposition 4.3. The following holds

$$
\lim _{k \rightarrow \infty} \alpha_{t}^{M E}=\alpha_{t}^{M}, \quad \lim _{k \rightarrow \infty} a_{t}^{M E}=a_{t}^{M}
$$

The proof of Proposition 4.3 is reported in Appendix E. We also find the following asymptotics. In case when the cost $\xi$ of misvaluation tends to zero, the principal gives full incentives to the agent to misvaluate, and the effort level will also be maximal.

$$
\lim _{\xi \rightarrow 0} \alpha_{t}^{M E}=1, \quad \lim _{\xi \rightarrow 0} a_{t}^{M E}=\infty, \quad \lim _{\xi \rightarrow 0} u_{t}^{M E}=\frac{\epsilon}{k}
$$

The expressions for $\alpha_{t}^{M E}$ and $a_{t}^{M E}$ correspond to the ones obtained in Capponi et al. (2011) in case of zero penalty for misvaluation. If, instead, there is zero penalty for effort, we obtain

$$
\begin{aligned}
\lim _{k \rightarrow 0} \alpha_{t}^{M E} & =1 \\
\lim _{k \rightarrow 0} a_{t}^{M E} & =\frac{\epsilon}{\sigma \xi}\left[\epsilon \operatorname{sech}\left(\frac{\epsilon T}{\sigma}\right) \sinh \left(\frac{\epsilon t}{\sigma}\right)-(\epsilon-1) \tanh \left(\frac{\epsilon t}{\sigma}\right)\right] \\
\lim _{k \rightarrow 0} u_{t}^{M E} & =\infty
\end{aligned}
$$


Here, the expressions for $\alpha_{t}^{M E}$ and $u_{t}^{M E}$ agree with their counterparts in the HolmstromMilgrom model with zero penalty for effort. The proofs of Eq. (4.31) and (4.32) are provided in Appendix $\mathrm{F}$.

\section{The contracting model when market anticipates effort and misvaluation}

We consider the case when the market correctly anticipates both the optimal effort and the misvaluation amount applied by the agent. This means that $\tilde{u}_{t}=u_{t}$, and $\tilde{a}_{t}=a_{t}$, and the market values the firm using $\hat{x}^{u, a}$.

We use $u_{t}^{H N}, a_{t}^{H N}, \alpha_{t}^{H N}$ to denote, respectively, the optimal effort, misvaluation, and payper-performance sensitivity. Here $H N$ stands as an abbreviation for Holmstrom-Milgrom with noise, in anticipation of the fact (proven later in the section) that, under this informational assumptions, the framework reduces to a Holmstrom-Milgrom setting, inclusive of noisy output signals.

\subsection{The agent}

Under the above choices of $\tilde{u}_{t}$ and $\tilde{a}_{t}$, we have that the Lagrangian (3.6) reduces to

$$
\mathcal{L}\left(t, u_{t}, a_{t}\right)=\alpha_{t}\left(u_{t} \epsilon-\frac{\gamma_{1}}{2} \alpha_{t} \frac{v_{t}^{2}}{\sigma^{2}}\right)-\left(\frac{k}{2} u_{t}^{2}+\frac{\xi}{2} a_{t}^{2}\right)
$$

It is then readily seen that the Lagrangian is maximized when

$$
u_{t}^{H N}=\frac{\epsilon \alpha_{t}}{K} \quad a_{t}^{H N}=0
$$

In particular, the agent never finds it optimal to misvalue the firm. This is intuitive, because the market would always value the firm at its fundamentals, and thus the agent would only incur costs without any benefit. As the only action that the agent applies is effort, such a scenario coincides with the Holmstrom-Milgrom setup in presence of noisy output values. Indeed, as we show later, we recover the results of Holmstrom and Milgrom (1987), when the noise intensity converges to zero.

\subsection{The principal}

Under the above choices of $\tilde{u}_{t}$ and $\tilde{a}_{t}$, we have that the functional optimized by the principal in Eq. (3.8), reduces to

$$
\mathcal{A}\left(t, \alpha_{t}\right)=\int_{0}^{T}\left[\epsilon u_{t}^{H N}-\frac{v_{t}^{2}}{2 \sigma^{2}}\left(\gamma_{2}\left(1-\alpha_{t}\right)^{2}+\gamma_{1} \alpha_{t}^{2}\right)\right] d t-\frac{1}{2} \int_{0}^{T} k\left(u_{t}^{H N}\right)^{2} d t
$$


Using the expression for $u_{t}^{H N}$ given in Eq. (5.2), it is readily seen that $\mathcal{A}$ is maximized if

$$
\alpha_{t}^{H N}=\frac{\frac{\epsilon^{2}}{K}+\frac{v_{t}^{2}}{\sigma^{2}} \gamma_{2}}{\frac{\epsilon^{2}}{K}+\frac{v_{t}^{2}}{\sigma^{2}}\left(\gamma_{1}+\gamma_{2}\right)}
$$

The above expression provides the pay-per-performance sensitivity in a Holmstrom-Milgrom type model with noisy output signals. As the noise intensity $\sigma$ gets smaller, we obtain the expected result

$$
\lim _{\sigma \rightarrow 0} \alpha_{t}^{H N}=\alpha_{t}^{H M}
$$

where we recall that $\alpha_{t}^{H M}$ has been defined in Eq. (4.25).

\section{Comparative Statics}

This section analyzes the sensitivity of optimal $\alpha_{t}, a_{t}$ and $u_{t}$ with respect to the various parameters of the model. Furthermore, we compare those quantities with their counterparts in the models where only misvaluation or effort is present, see Capponi et al. (2011) and Holmstrom and Milgrom (1987) respectively. We first summarize the main findings and then separately analyze these sensitivities. We find that, in case when the market is unsophisticated, the agent is optimally induced to apply both effort and to overvalue the firm. As such, the pay-per-performance sensitivity of the contract is higher than in the case where he can only be induced to apply one of those actions. When market investors are unsophisticated, we find that the amount of misvaluation increases over time, see Figure 1. This is consistent with the statements of Jensen (2004), who claims that if managers have troubles meeting the estimates for a year, they push expenses forward. He then claims that revenues borrowed from the future and today's expenses pushed to tomorrow require even more manipulation in the future to "forestall the day of reckoning".

We also find that the principal will induce the agent to apply a higher level of effort, relative to what she would do in the Holmstrom-Milgrom model. Similarly, she will induce the agent to overvalue the firm more relative to the contracting framework in which she can only induce misvaluation. Moreover, as it can be seen in Figure 4, if effort and misvaluation are equally costly, most of the incentives provided by the principal are to induce the agent to misvalue, unless the accounting noise becomes very large, in which case he is given more incentives to apply effort. An analysis of the joint dependence of effort and misvaluation from the two cost factors shows that the misvaluation cost mainly determines the misvaluation amount, while the effort action is mainly affected by the effort cost.

If the market is sophisticated and able to correctly anticipate both dimensions of the moral hazard, the principal only needs to provide the agent incentives to apply effort. Similarly to the basic HM model, those incentives decrease as the cost for effort increases. However, as evidenced in Figure 6, higher incentives need to be provided by the agent when output signals are noisy. Indeed, the right panel of Figure 6] shows that, as the effort cost gets larger, a significant portion of incentives is provided to compensate for accounting noise. We also find that the pay-per-performance sensitivity increases as the level of accounting noise increases. 
Indeed, as evidenced in Figure 2, for very small levels of accounting noise, the incentives provided in the models where misvaluation can be applied are always higher than the ones provided in the model where the agent is only induced to apply effort. However, as the noise intensity increases, the incentives provided in the model where only effort can be applied become higher than the ones provided in the model where only misvaluation can be applied. This may happen because, as $\sigma$ gets larger, the impact of misvaluation on the market price of the firm becomes smaller and smaller. Indeed, as it can be checked from Eq. (2.10), for a fixed level $a_{t}$ of misvaluation, $\hat{x}_{t}^{u, 0} \rightarrow \hat{x}_{t}^{u, a}$ as $\sigma \rightarrow \infty$. This is also reflected in the graphs of Fig, 2 .

In all graphs below, we set the time horizon at $T=1$ year. We investigate the behavior of the optimal $a_{t}, u_{t}$, and $\alpha_{t}$ at time $t=0.5$. In the legends, we use "M" to denote the contracting framework, only inclusive of misvaluation, analyzed in Capponi et al. (2011). We then use "ME" to denote the contracting model obtained when the market only anticipates effort, and "HN" to denote the one obtained when the market anticipates both effort and misvaluation. We then use "HM" to denote the Holmstrom-Milgrom model.

\subsection{Sensitivity Analysis with respect to time}

The PPS in the model in which both effort and misvaluation are present is U-shaped, and contrasts with the one obtained in the contracting model with only misvaluation, where it is always increasing. In both cases, the optimal amount is negligible initially, because the actual output value is assumed to be known at time zero, and thus there is no benefit from misvaluation. Initially, the principal gives full incentives to the agent, aimed primarily at inducing him to apply effort, rather than misvaluation. As time goes by, the principal wants the agent to apply a higher level of misvaluation, while not as much effort is needed, and the pay-per-performance sensitivity is decreased. However, later on, it is optimal to have the agent apply increasingly higher effort. To this purpose, the principal increases again the pay-per-performance sensitivity to keep the levels of both misvaluation and effort increasing.

Moreover, we can see that the PPS in contracting models in which misvaluation is allowed is always higher than the (constant) PPS obtained in the Holmstrom-Milgrom model.

\subsection{Sensitivity Analysis with respect to $\sigma$}

In this part, we study the behavior of optimal pay-per-performance sensitivity, optimal misvaluation and effort with respect to $\sigma$.

\subsubsection{Optimal pay-per-performance sensitivity versus $\sigma$}

Figure 2 shows that while the optimal pay-per-performance sensitivity is decreasing in $\sigma$ in absence of effort, it may exhibit different patterns when effort is present. As $\sigma$ increases, misvaluation yields smaller benefits, and consequently the PPS in the model where only misvaluation is allowed decreases. On the other hand, Figure 2 shows that as $\sigma$ gets larger, the principal needs to provide extra incentivies to the agent to apply effort, if the market correctly antipate both dimensions of the moral hazard. This explains why in the model 

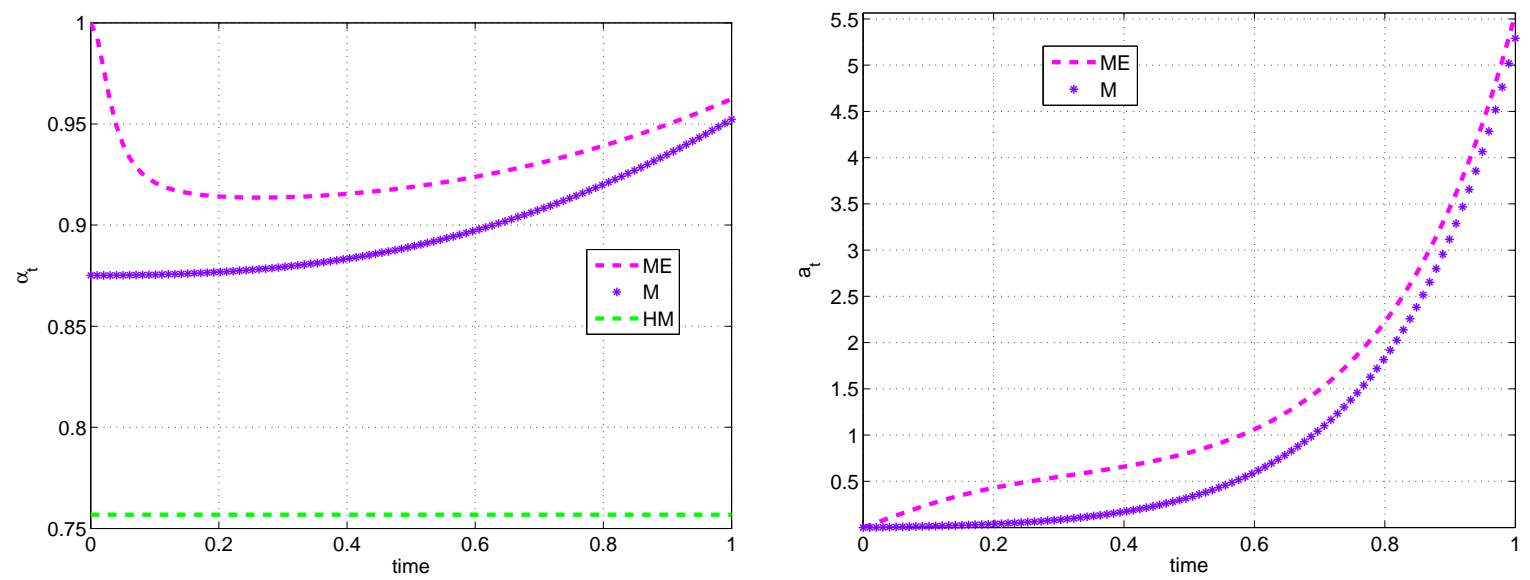

Figure 1: The parameters common to all graphs are $\sigma=0.2, \epsilon=1, \xi=0.9, k=1.2, \gamma_{1}=0.3$, $\gamma_{2}=0.1$. The left graph refers to the pay-per-performance sensitivity over time, while the right graph to the misvaluation amount over time.

where investors are unsophisticated, the optimal PPS is not necessarily monotone. We next zoom on that. If the cost for misvaluation is high and the effort penalty small, the agent is more resilient to misvalue the output value, and prefers to increase it with effort. Additionally, as discussed above, the higher $\sigma$, the higher the incentive for the principal to induce agent to apply effort relative to misvaluation. This parametric case is illustrated in the top left graph of Figure 2, where we can see that $\alpha_{t}$ monotonically increases with $\sigma$. When effort has significantly higher penalty than misvaluation, the principal knows that it is costly for the agent to apply effort, and therefore she offers her smaller incentives to do so, unless $\sigma$ becomes large, and really needs the agent's effort to extract benefits, see bottom right graph.

\subsubsection{Optimal misvaluation versus $\sigma$}

Figure 3 shows that if investors are unsophisticated and effort can be applied, the principal always induces the agent to apply higher misvaluation with respect to the case where no effort can be applied. If investors are unsophisticated, the mean-variance tradeoff $\epsilon$ is large enough, and the penalty for misvaluation is significantly smaller than the penalty for effort, then the principal may have incentives to induce the agent to apply higher misvaluation for larger values of $\sigma$, as long as $\sigma$ does not become too large. In other words, the relation between $a_{t}$ and $\sigma$ may not be monotone, as seen in the case of the bottom right graph of Figure 3 ,

\subsection{Sensitivity Analysis with respect to $\epsilon$}

Here, we investigate the behavior of optimal pay-per-performance sensitivity, optimal misvaluation and optimal effort with respect to $\epsilon$. 

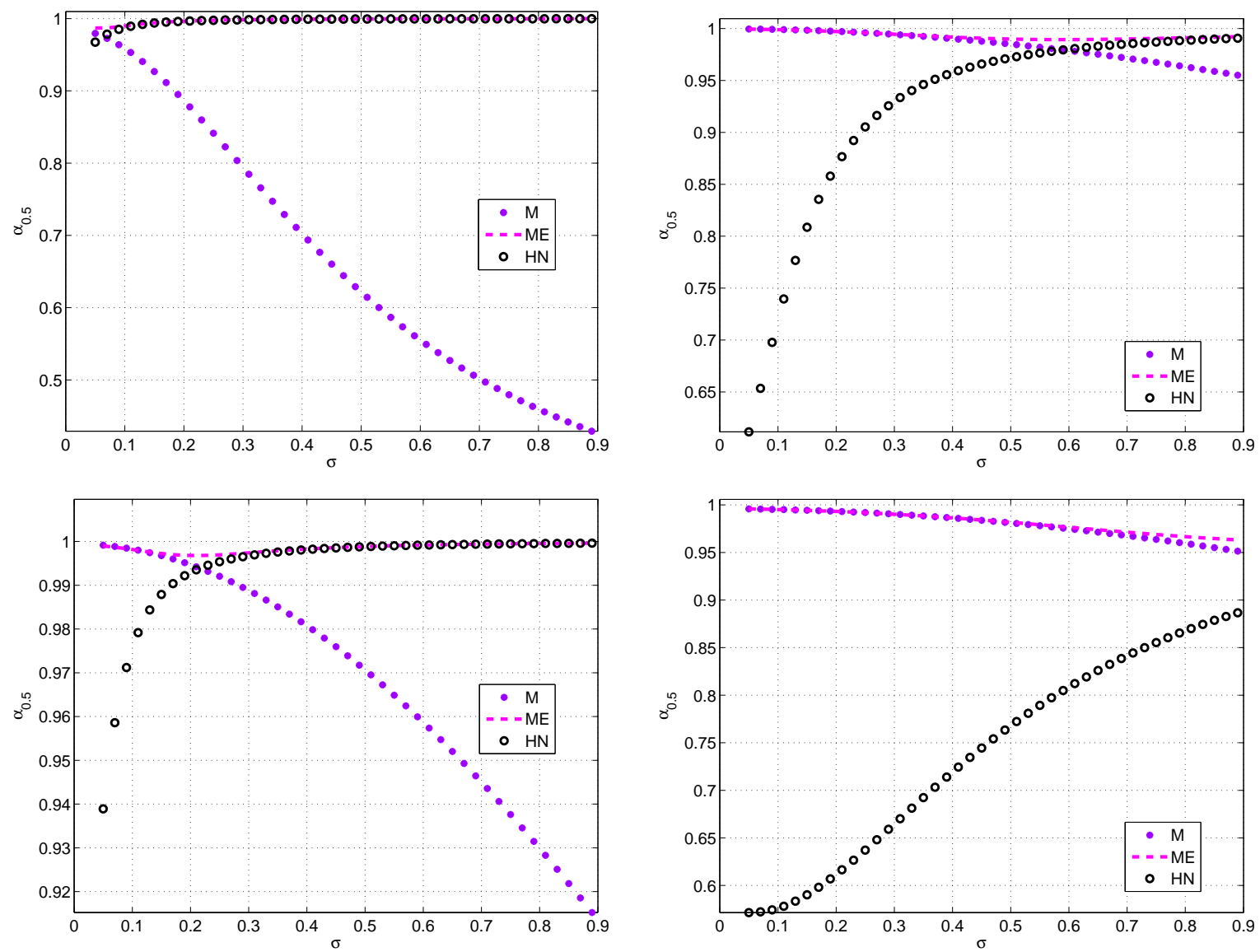

Figure 2: The parameters common to all graphs are $\gamma_{1}=0.3, \gamma_{2}=0.1$. Each graph plots $\alpha_{t}$ versus $\sigma$, for a specific triple $(k, \xi, \epsilon)$ of effort and misvaluation cost. Top left graph: $(k, \xi, \epsilon)=(0.2,10,0.1)$. Top right graph: $(k, \xi, \epsilon)=(10,0.2,0.1)$. Bottom left graph: $(k, \xi, \epsilon)=(0.4,0.4,0.1)$. Bottom right graph: $(k, \xi, \epsilon)=(10,0.2,0.4)$. 

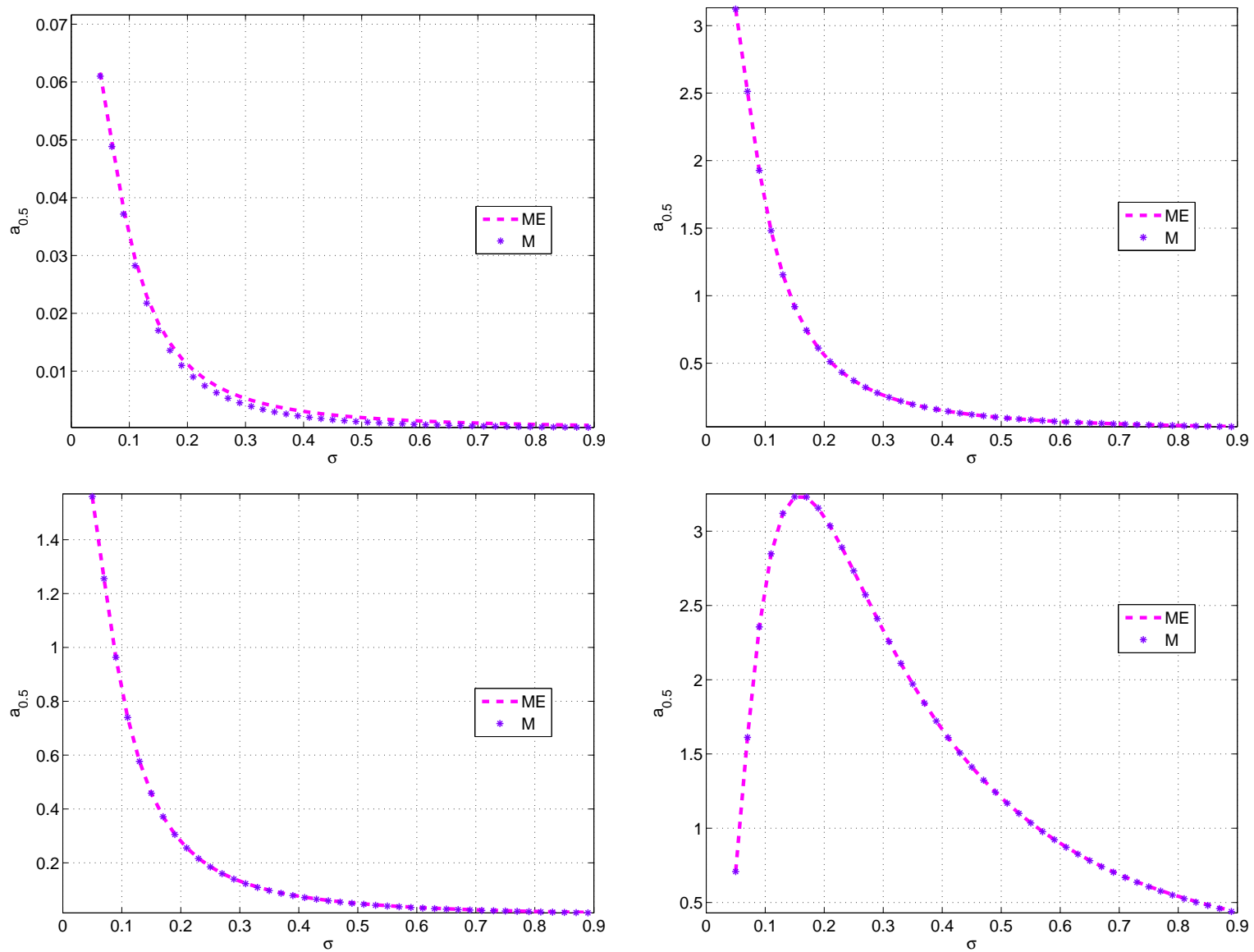

Figure 3: The parameters common to all graphs are $\gamma_{1}=0.3, \gamma_{2}=0.1$. Each graph plots the misvaluation action $a_{t}$ versus $\sigma$, for a specific triple $(k, \xi, \epsilon)$. Top left graph: $(k, \xi, \epsilon)=(0.2,10,0.1)$. Top right graph: $(k, \xi, \epsilon)=(10,0.2,0.1)$. Bottom left graph: $(k, \xi, \epsilon)=(0.4,0.4,0.1)$. Bottom right graph: $(k, \xi, \epsilon)=(10,0.2,0.4)$. 

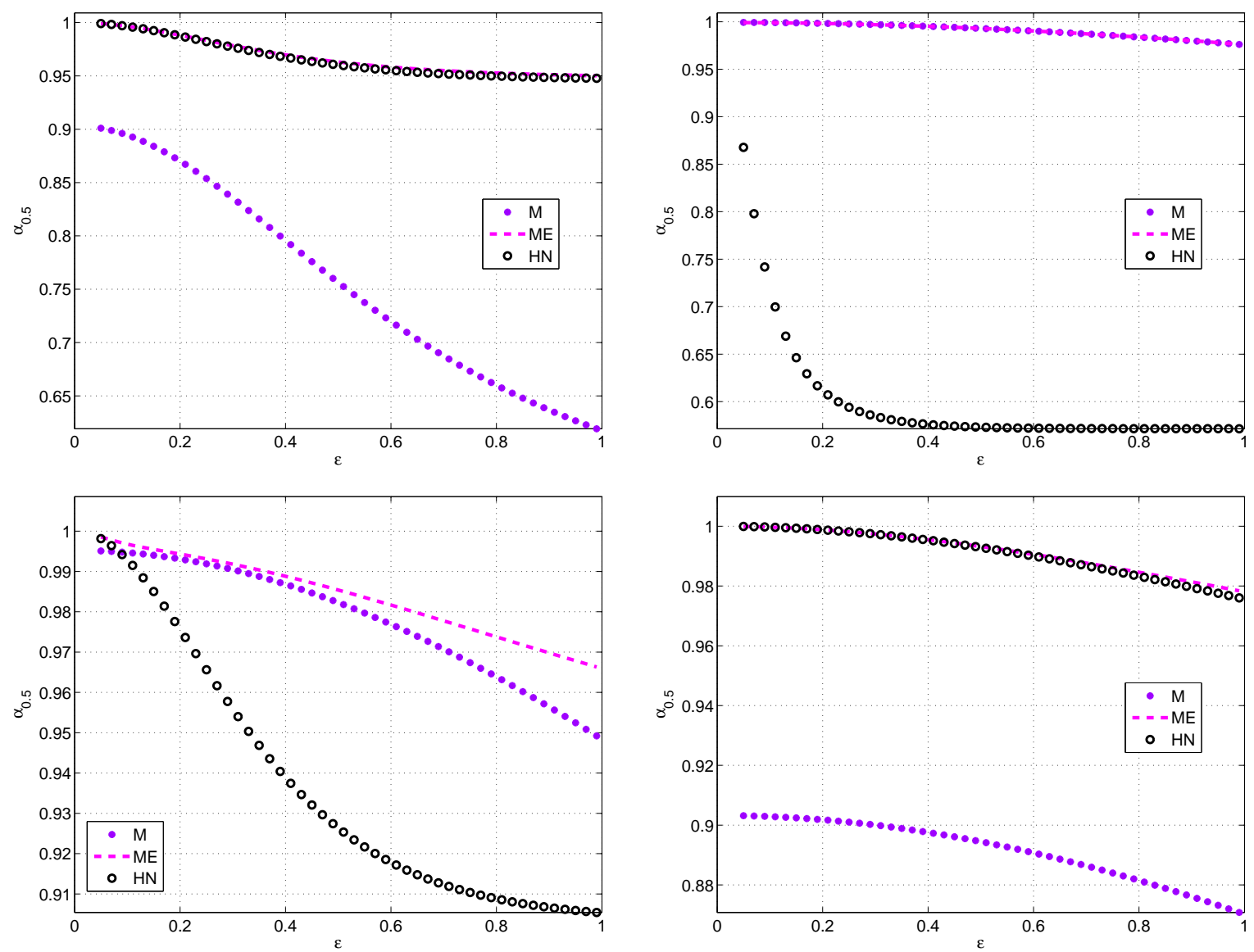

Figure 4: The parameters common to all graphs are $\gamma_{1}=0.3, \gamma_{2}=0.3$. Each graph plots $\alpha_{t}$ versus $\epsilon$, for a specific triple $(k, \xi, \sigma)$. Top left graph: $(k, \xi, \sigma)=(0.2,10,0.2)$. Top right graph: $(k, \xi, \sigma)=(10,0.2,0.1)$. Bottom left graph: $(k, \xi, \sigma)=(0.4,0.4,0.1)$. Bottom right graph: $(k, \xi, \sigma)=(0.4,0.4,1)$. 


\subsubsection{Optimal pay-per-performance sensitivity versus $\epsilon$}

We see from Figure 4 that the pay-per-performance sensitivity $\alpha_{t}$ is decreasing in $\epsilon$, regardless of whether the market consists of unsophisticated or sophisticated investors who correctly anticipate misvaluation. This contrasts with Figure 2, where the principal typically increases the incentives when the noise volatility $\sigma$ is large enough. This may be explained observing that, for a contract consisting of cash and of performance related compensation depending linearly on the market estimate, increasing $\epsilon$ is equivalent to increasing portfolio holdings of the agent in the risky asset relative to cash. This interpretation helps understanding why high $\alpha$ and high $\epsilon$ have the same effect on the agent, and result in the agent having higher exposure to the risky part of the compensation. Therefore, if $\epsilon$ goes up, then $\alpha_{t}$ should go down to compensate for that. Interestingly, higher incentives for effort are given when either the cost for misvaluation or the intensity of accounting noise is large (top left and bottom right graph), whereas higher incentives for misvaluation are provided when the effort penalty is large or the accounting noise intensity small (top right and bottom left graph).

\subsubsection{Optimal misvaluation action versus $\epsilon$}

Figure [5] shows that the principal does not necessarily induce the agent to misvalue more when $\epsilon$ increases. The dependence of $a_{t}$ on $\epsilon$ is generally inverted U-shaped, except when $\sigma$ is very large as in the right bottom graph, where it becomes increasing.

\subsection{Sensitivity Analysis with respect to effort and misvaluation costs}

In this part, we analyze the dependence of optimal pay-per-performance sensitivity, misvaluation and effort on the costs incurred when taking the actions.

\subsubsection{Optimal pay-per-performance sensitivity versus $k$ and $\xi$}

From the top graphs of Figure 6 we note the usual pattern, the optimal pay-per-performance sensitivity is always higher than in the cases in which only one of the two actions is allowed. Moreover, the two top graphs show that, in the ME model, PPS has similar sensitivity with respect to the increases in cost of misvaluation (for a fixed effort penalty $k$ ), and to the increases in effort penalty (for a fixed misvaluation cost $\xi$ ). The graphs also indicate that the principal provides higher incentives in the model in which only misvaluation is present, relative to the model in which only effort can be applied.

The bottom graph, which analyzes the joint dependence of $\alpha_{t}$ on misvaluation and effort, reinforces previous statements, showing that for any given level of effort (misvaluation) cost, the pay-per-performance sensitivity increases to one as the misvaluation (effort) cost decreases to zero. In other words, the whole firm is transferred to the agent if he is willing to apply an infinite amount of effort or misvaluation. 

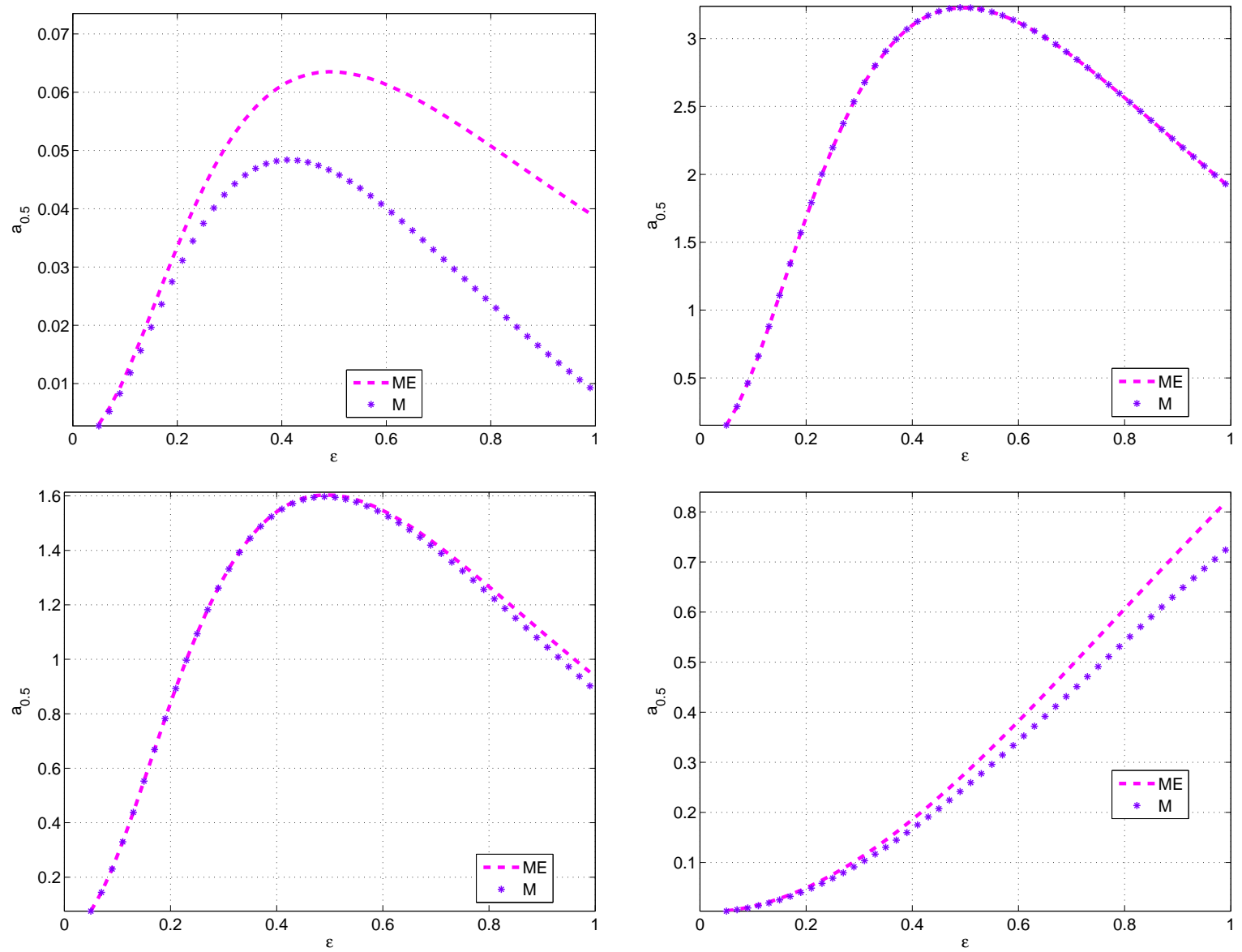

Figure 5: The parameters common to all graphs are $\gamma_{1}=0.3, \gamma_{2}=0.3$. Each graph plots $a_{t}$ versus $\epsilon$, for a specific triple $(k, \xi, \sigma)$. Top left graph: $(k, \xi, \sigma)=(0.2,10,0.2)$. Top right graph: $(k, \xi, \sigma)=(10,0.2,0.1)$. Bottom left graph: $(k, \xi, \sigma)=(0.4,0.4,0.1)$. Bottom right graph: $(k, \xi, \sigma)=(0.4,0.4,1)$. 

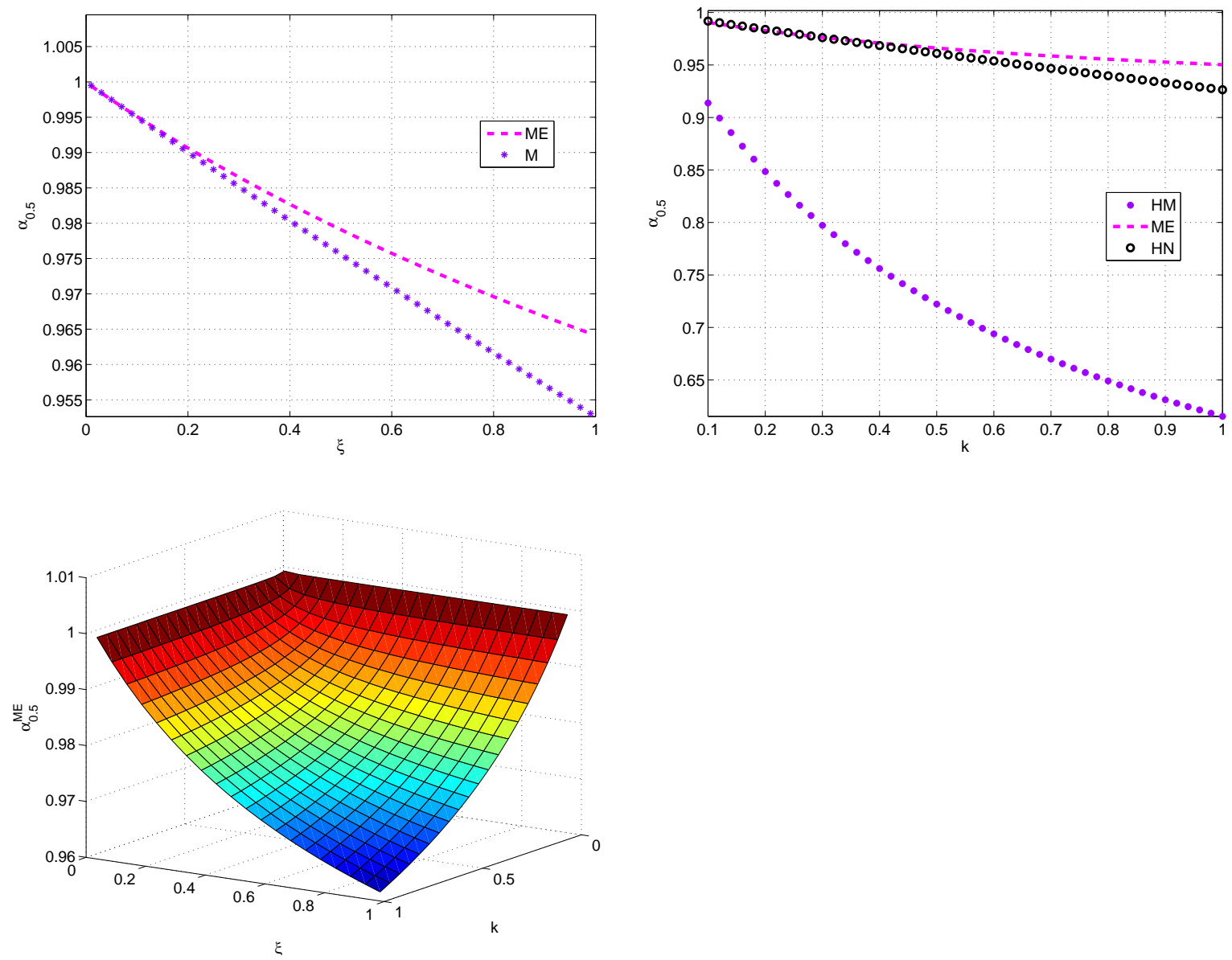

Figure 6: The parameters used for the top graphs are $\epsilon=0.2, \sigma=0.1, \gamma_{1}=1, \gamma_{2}=0.3$. Top left graph: $\alpha_{t}$ versus $\xi$ for a level of effort penalty $k=0.2$. Top right graph: $\alpha_{t}$ versus $k$ when the cost of misvaluation is $\xi=0.2$. The bottom graph plots the pay-per-performance sensitivity $\alpha_{t}$, with respect to both $k$ and $\xi$. The parameters used for the bottom graph are $\epsilon=0.2, \sigma=0.3, \gamma_{1}=0.8, \gamma_{2}=0.4$. 

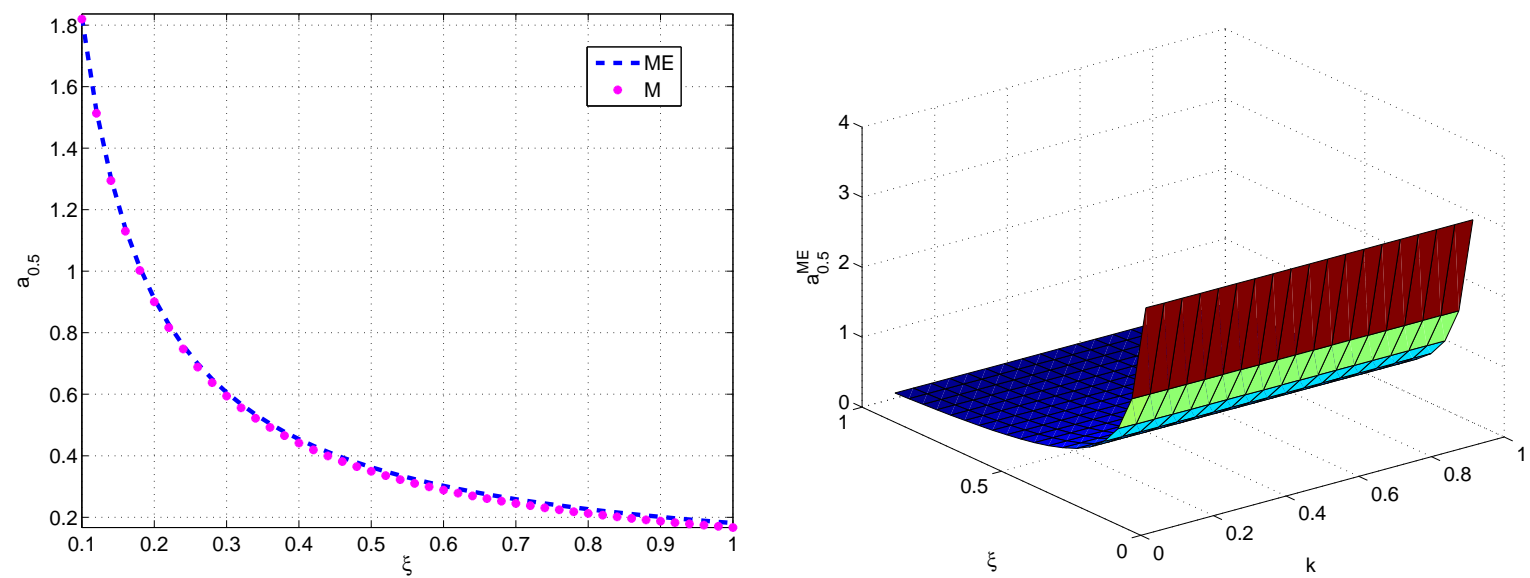

Figure 7: The left graph plots $a_{t}$ versus $\xi$. The parameters used are $\epsilon=0.2, k=0.2, \sigma=0.3$, $\gamma_{2}=0.6$, and $T=1$. The right graph plots the misvaluation $a_{t}$, jointly with respect to $k$ and $\xi$ under the parameter choice $\epsilon=0.2, \sigma=0.3, \gamma_{1}=0.8, \gamma_{2}=0.4$.

\subsubsection{Optimal misvaluation action versus $k$ and $\xi$}

Figure [ 7 shows that the level of misvaluation decreases as the penalty $\xi$ gets larger. Moreover, the misvaluation is mainly driven by the size of $\xi$, that is, for a given level of $\xi$, it exhibits negligible variations with respect to the effort penalty $k$.

\subsubsection{Optimal effort versus $k$ and $\xi$}

Figure 8 shows that the amount of effort decreases as the penalty $k$ for effort increases. The effort choice is mainly driven by the cost $k$, and it exhibits negligible variations with respect to $\xi$.

\subsection{Sensitivity Analysis with respect to the level of risk aversions}

In this part, we analyze the dependence of optimal pay-per-performance sensitivity, optimal misvaluation and optimal effort on the level of risk aversions of principal and agent.

\subsubsection{Optimal pay-per-performance sensitivity versus $\gamma_{1}$ and $\gamma_{2}$}

Figure 9 shows that the pay-per-performance sensitivity is decreasing in the risk aversion level $\gamma_{1}$ of the agent. This is because when the agent does not like risk, the incentives are less efficient. We also notice that pay-per-performance sensitivity is increasing in the risk aversion level of the principal in all cases. This is because with higher $\gamma_{2}$, the principal dislikes risk more, and wants to transfer it to the agent. 

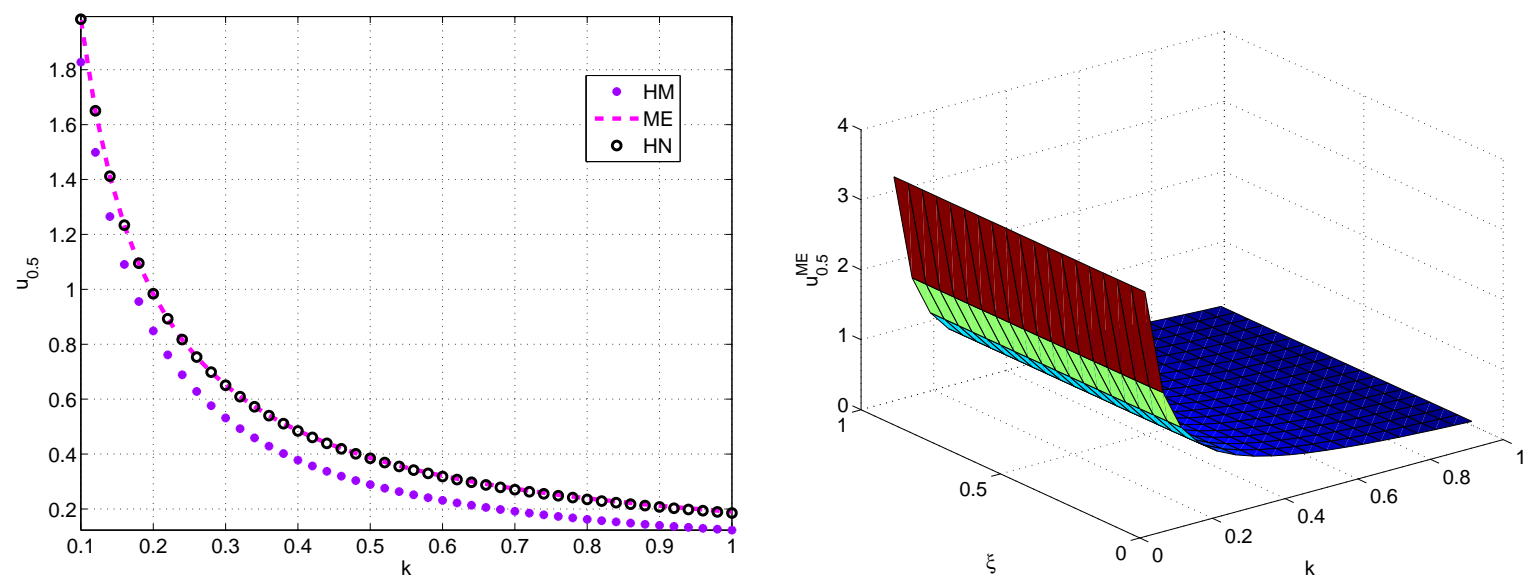

Figure 8: The left graph plots $u_{t}$ versus $k$. The parameters used are $\xi=0.2, \epsilon=0.2, \sigma=0.3$, $\gamma_{1}=1, \gamma_{2}=0.6$. The right graph plots the effort $u_{t}$, jointly with respect to $k$ and $\xi$ under the parameter choice $\epsilon=0.2, \sigma=0.3, \gamma_{1}=0.8, \gamma_{2}=0.4$.
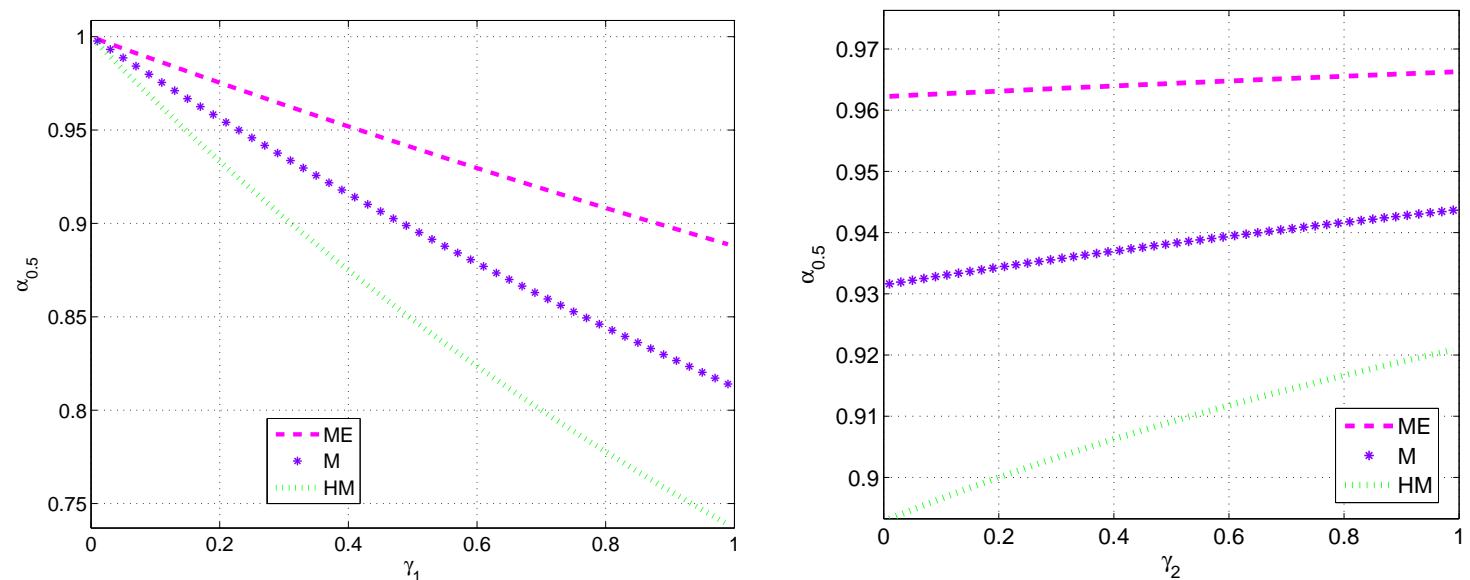

Figure 9: The parameters common to both graphs are $\epsilon=1, \xi=0.4, \sigma=0.4, k=0.4, T=1$. The left graph plots $\alpha_{t}$ versus $\gamma_{1}$ for a given level of principal's risk aversion $\gamma_{2}=0.3$. The right graph plots $\alpha_{t}$ versus $\gamma_{2}$ for a given level of agent risk aversion $\gamma_{1}=0.3$. 

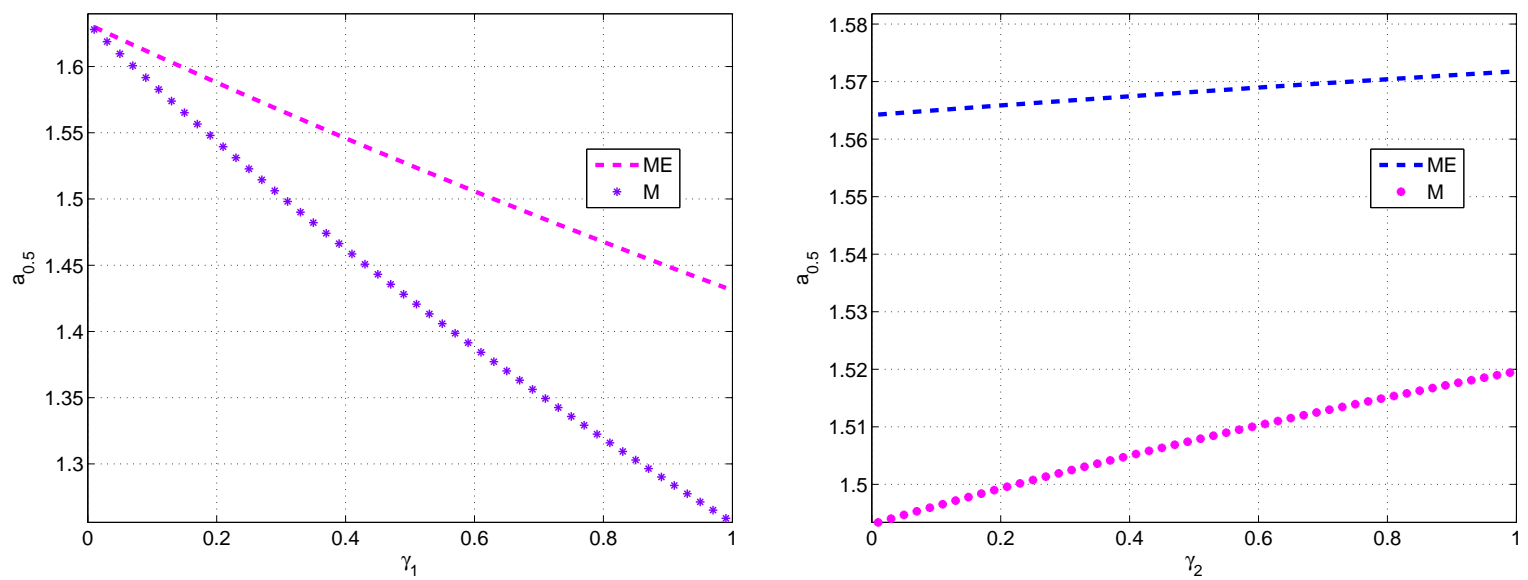

Figure 10: The parameters common to both graphs are $\epsilon=1, \xi=0.4, \sigma=0.4, k=0.4, T=1$. The left graph plots $a_{t}$ versus $\gamma_{1}$ for a given level of principal's risk aversion $\gamma_{2}=0.3$. The right graph plots $a_{t}$ versus $\gamma_{2}$ for a given level of agent risk aversion $\gamma_{1}=0.3$.

\subsubsection{Optimal misvaluation action versus $\gamma_{1}$ and $\gamma_{2}$}

We see from Figure 10 that, when the market is unsophisticated, the amount of misvaluation decreases with $\gamma_{1}$. This is because a more risk averse agent gets a lower percentage of the firm's output, and is less inclined to misvalue the fundamental value. Similarly, since everything is driven by the relative size of risk aversions, the amount of induced misvaluation increases if the principal is more risk averse. More precisely, the higher the risk aversion of the principal, the higher percentage of the firm she gives to the agent, who then has higher incentives to misvalue.

\subsubsection{Optimal effort versus $\gamma_{1}$ and $\gamma_{2}$}

The behavior of the effort action follows a similar pattern to the misvaluation action, and for the same reasons. More precisely, from Figure 11, we see that the amount of effort decreases with $\gamma_{1}$. Similarly, the higher the risk aversion of the principal, the higher the agent's effort. This is in agreement with all the standard contracting models a la Holmstrom-Milgrom.

\section{Conclusions}

This paper provides a general contracting framework in which there are two possible sources of moral hazard, namely effort and misvaluation, and output signals are noisy. We have studied optimal linear contracts offered by the principal to the agent, which are contingent on market price estimates computed by a third player, the market. The latter may be unable to reconstrct the optimal level of effort and misvaluation applied by the agent, due to imperfect knowledge of principal and agent objectives, and therefore computes the price based on its perceived level of effort and misvaluation. We have then specialized our framework to deal with two economic scenarios, both of which assuming that the market can correctly reconstruct the 

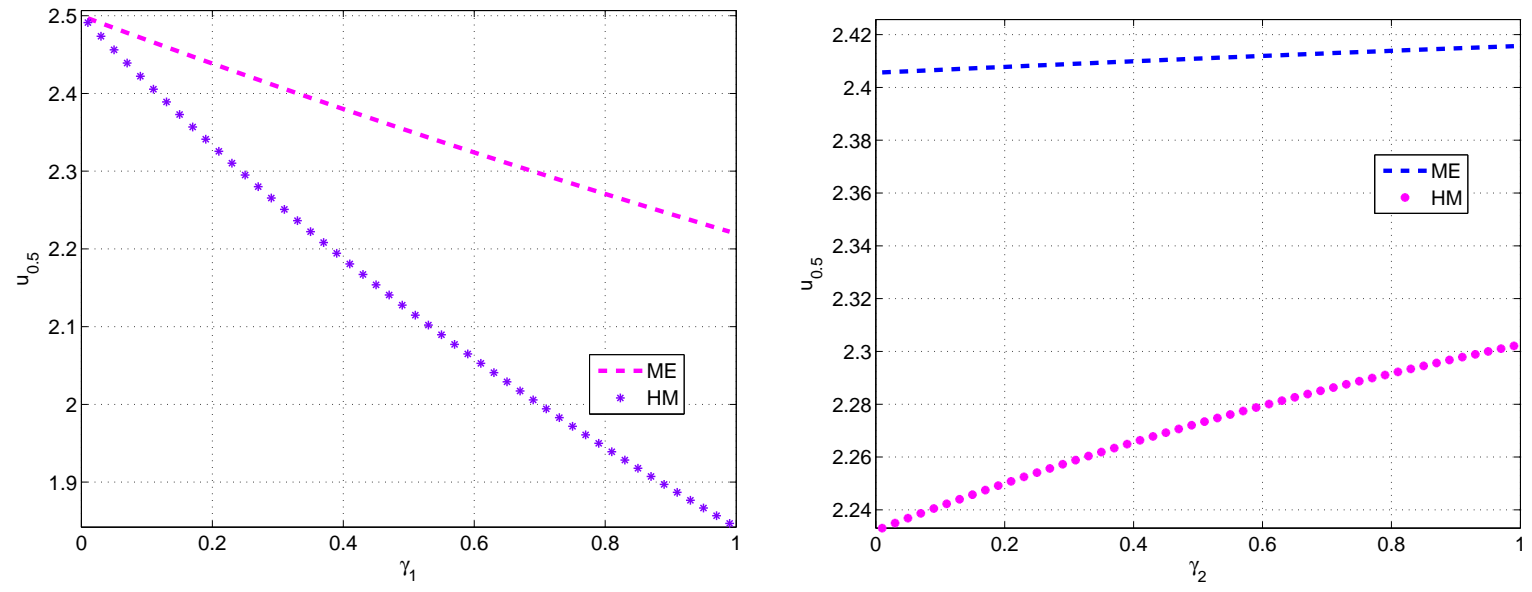

Figure 11: The parameters common to both graphs are $\epsilon=1, \xi=0.4, \sigma=0.4, k=0.4, T=1$. The left graph plots $u_{t}$ versus $\gamma_{1}$ for a given level of principal's risk aversion $\gamma_{2}=0.3$. The right graph plots $u_{t}$ versus $\gamma_{2}$ for a given level of agent risk aversion $\gamma_{1}=0.3$.

optimal effort level of the agent. The first scenario assumes that the market is composed of unsophisticated investors and that both principal and agent have a short-term horizon. In this time frame, the market is unable to detect that the agent is altering the fundamental value of the firm. We found semi-analytical expressions for the optimal pay-per-performance sensitivity, effort, and misvaluation amount, after solving a singular second order differential equation coming from a variational formulation. The second scenario, instead, considers a sophisticated market, consisting of investors who can correctly detect misvaluation.

We develop a thorough comparative statics analysis to analyze the interplay between effort and misvaluation, as well as the dependence of the actions and of the optimal contract on the various model parameters. In the first scenario, we found that misvaluation increases over time. Moreover, the optimal compensation exhibits higher pay-per-performance sensitivity relative to a framework in which the agent is only compensated for effort, or only compensated for misvaluation. Therefore, when market is unsophisticated, the fact that shareholders pay a significant fraction of the compensation to induce the manager to misvalue indicates that transferring more power to shareholders would have negative consequences. Rather, more frequent auditing, captured by the misvaluation cost factor, is needed in order to alleviate the concerns about corporate oversight. On the other side, when the market is sophisticated and anticipates misvaluation, we found that zero misvaluation occurs in equilibrium, as it is never in the interest of the agent to do so. Therefore, such a framework reduces to a HolmstromMilgrom type model with the only difference being that the output is noisy. Interestingly, we found that the agent requires a higher PPS, as the intensity of the accounting noise increases.

In the future, we would like to exploit the full power of the model, and study other special cases corresponding to different choices of the perceived level of effort, and misvaluation of the agent. We would also like to incorporate auditing, or other oversight measures, into our framework. 


\section{A Proofs related to Section 2}

We prove Lemma 2.1 first.

Proof. Note that Eq. (2.5) gives

$$
d \hat{x}_{s}^{\tilde{u}, \tilde{a}}=\epsilon \tilde{u}_{s} d t+\frac{v_{s}}{\sigma} d \hat{Z}_{s}^{\tilde{u}, \tilde{a}}
$$

which, multiplied with $\frac{\sigma}{v_{s}}$ and integrated from 0 to $t$, gives (2.8). Using Eq. (2.6), we obtain

$$
\begin{aligned}
& \hat{Z}_{t}^{u, a}=Z_{t}^{a}+\frac{1}{\sigma} \int_{0}^{t} \frac{x_{s}-\hat{x}_{s}^{u, a}}{\sigma} d s \\
& \hat{Z}_{t}^{\tilde{u}, \tilde{a}}=Z_{t}^{\tilde{a}}+\frac{1}{\sigma} \int_{0}^{t} \frac{x_{s}-\hat{x}_{s}^{\tilde{u}, \tilde{a}}}{\sigma} d s
\end{aligned}
$$

leading to

$$
\hat{Z}_{t}^{u, a}-\hat{Z}_{t}^{\tilde{u}, \tilde{a}}=Z_{t}^{a}-Z_{t}^{\tilde{a}}+\int_{0}^{t} \frac{\hat{x}_{s}^{\tilde{u}, \tilde{a}}-\hat{x}_{s}^{u, a}}{\sigma} d s
$$

Using the definition of $Z_{t}^{a}$ given in Eq. (2.2) we obtain Eq. (2.9) after a straightforward rearrangement.

We prove Lemma 2.2 next.

Proof. Let us denote

$$
\omega_{t}=\hat{x}_{t}^{\tilde{u}, \tilde{a}}-\hat{x}_{t}^{u, a}
$$

Using Eq. (2.9), we obtain upon differentiation that

$$
\frac{d}{d t}\left[\hat{Z}_{t}^{u, a}-\hat{Z}_{t}^{\hat{u}, \hat{a}}\right]=\frac{\tilde{a}_{t}-a_{t}}{\sigma}+\frac{\hat{x}_{t}^{\tilde{u}, \tilde{a}}-\hat{x}_{t}^{u, a}}{\sigma}
$$

or, equivalently,

$$
\hat{x}_{t}^{\tilde{u}, \tilde{a}}-x_{t}^{u, a}=\sigma \frac{d}{d t}\left[\hat{Z}_{t}^{u, a}-\hat{Z}_{t}^{\tilde{u}, \tilde{a}}\right]+a_{t}-\tilde{a}_{t}
$$

From Eq. (2.5), we deduce

$$
\frac{d}{d t}\left(\hat{x}_{t}^{u, a}-\hat{x}_{t}^{\tilde{u}, \tilde{a}}\right)=\epsilon\left(u_{t}-\tilde{u}_{t}\right)+\frac{v_{t}}{\sigma} \frac{d}{d t}\left(\hat{Z}_{t}^{u, a}-\hat{Z}_{t}^{\tilde{u}, \tilde{a}}\right)
$$

Plugging Eq. (A.6) into Eq. (A.5), we get

$$
\hat{x}_{t}^{\tilde{u}, \tilde{a}}-\hat{x}_{t}^{u, a}=\sigma\left(-\frac{\sigma}{v_{t}} \frac{d}{d t}\left(\hat{x}_{t}^{\tilde{u}, \tilde{a}}-\hat{x}_{t}^{u, a}\right)-\frac{\sigma}{v_{t}} \epsilon\left(u_{t}-\tilde{u}_{t}\right)\right)+a_{t}-\tilde{a}_{t}
$$

From the definition of $\omega_{t}$ in Eq. (A.4), and Eq. (A.7), we get the ordinary differential equation

$$
\omega_{t}+\frac{\sigma^{2}}{v_{t}} \omega_{t}^{\prime}=\epsilon \frac{\sigma^{2}}{v_{t}}\left(\tilde{u}_{t}-u_{t}\right)+\left(a_{t}-\tilde{a}_{t}\right)
$$


By definition of $v_{t}$, we have that $\frac{\sigma^{2}}{v_{t}}=\frac{\sigma}{\epsilon} \frac{\cosh \left(\frac{\epsilon t}{\sigma}\right)}{\sinh \left(\frac{\epsilon t}{\sigma}\right)}$. Multiplying both sides of Eq. (A.8) by $\frac{\epsilon}{\sigma} \sinh \left(\frac{\epsilon t}{\sigma}\right)$, we obtain

$$
\frac{\epsilon}{\sigma} \sinh \left(\frac{\epsilon t}{\sigma}\right) \omega_{t}+\cosh \left(\frac{\epsilon t}{\sigma}\right) \omega_{t}^{\prime}=\epsilon \cosh \left(\frac{\epsilon t}{\sigma}\right)\left(\tilde{u}_{t}-u_{t}\right)+\frac{\epsilon}{\sigma} \sinh \left(\frac{\epsilon t}{\sigma}\right)\left(a_{t}-\tilde{a}_{t}\right)
$$

or, equivalently,

$$
\frac{d}{d t}\left(\cosh \left(\frac{\epsilon t}{\sigma}\right) w_{t}\right)=\epsilon \cosh \left(\frac{\epsilon t}{\sigma}\right)\left(\tilde{u}_{t}-u_{t}\right)+\frac{\epsilon}{\sigma} \sinh \left(\frac{\epsilon t}{\sigma}\right)\left(a_{t}-\tilde{a}_{t}\right)
$$

Integrating from 0 to t, we get Eq. (2.10).

\section{B Definitions and Theorems related to Section 3}

Definition B.1. (Tonelli Lagrangian) For $d \geq 1, L:[0, T] \times \mathbb{R}^{d} \times \mathbb{R}^{d} \mapsto \mathbb{R}$ is said to be a Tonelli Lagrangian if

(T1) L is a twice continuously differentiable function.

(T2) The Hessian matrix $\nabla_{\mathbf{v} \mathbf{v}} L(t, \mathbf{x}, \mathbf{v})$ of $L$ with respect to $\mathbf{v}$ is positive semidefinite for all $(t, \mathbf{x}, \mathbf{v}) \in[0, T] \times \mathbb{R}^{d} \times \mathbb{R}^{d}$. In other words, the map $\mathbf{v} \mapsto L(t, \mathbf{x}, \mathbf{v})$ is convex.

(T3) $L$ is coercive. In other words, there exist constants $m_{1}>0$ and $m_{2} \in \mathbb{R}$ such that $L(t, \mathbf{x}, \mathbf{v}) \geq m_{1}|\mathbf{v}|^{2}+m_{2}$ for all $(t, \mathbf{x}, \mathbf{v}) \in[0, T] \times \mathbb{R}^{d} \times \mathbb{R}^{d}$.

Definition B.2. (Tonelli Lagrangian) For $d \geq 1, L:[0, T] \times \mathbb{R}^{d} \times \mathbb{R}^{d} \mapsto \mathbb{R}$ is said to be a Tonelli Lagrangian if

(T1) L is a twice continuously differentiable function.

(T2) The Hessian matrix $\nabla_{\mathbf{v v}} L(t, \mathbf{x}, \mathbf{v})$ of $L$ with respect to $\mathbf{v}$ is positive semidefinite for all $(t, \mathbf{x}, \mathbf{v}) \in[0, T] \times \mathbb{R}^{d} \times \mathbb{R}^{d}$. In other words, the map $\mathbf{v} \mapsto L(t, \mathbf{x}, \mathbf{v})$ is convex.

(T3) $L$ is coercive. In other words, there exist constants $m_{1}>0$ and $m_{2} \in \mathbb{R}$ such that $L(t, \mathbf{x}, \mathbf{v}) \geq m_{1}|\mathbf{v}|^{2}+m_{2}$ for all $(t, \mathbf{x}, \mathbf{v}) \in[0, T] \times \mathbb{R}^{d} \times \mathbb{R}^{d}$.

Theorem B.2. (Tonelli's existence theorem) When (T1)-(T3) hold, then $\int_{0}^{T} L\left(t, \mathbf{x}_{t}, \mathbf{x}_{t}^{\prime}\right) d t$ has a minimizer $\mathbf{x}_{t}^{*}$ in the space of absolutely continuous functions. Moreover,

- If $(\mathbf{x}, \mathbf{v}) \mapsto L(t, \mathbf{x}, \mathbf{v})$ is convex, then the minimizer $\mathbf{x}_{t}^{*}$ Lipschitz continuous.

- If $\mathbf{v} \mapsto L(t, \mathbf{x}, \mathbf{v})$ is strictly convex, then the minimizer $\mathbf{x}_{t}^{*}$ is a twice continuously differentiable function on $[0, T]$. 
- If $(\mathbf{x}, \mathbf{v}) \mapsto L(t, \mathbf{x}, \mathbf{v})$ is strictly convex, then the minimizer $\mathbf{x}_{t}^{*}$ is unique.

Remark B.7. The condition that $(\mathbf{x}, \mathbf{v}) \mapsto L(t, \mathbf{x}, \mathbf{v})$ is strictly convex can be weakened to $(\mathbf{x}, \mathbf{v}) \mapsto L(t, \mathbf{x}, \mathbf{v})$ is convex and $\mathbf{v} \mapsto L(t, \mathbf{x}, \mathbf{v})$ is strictly convex to get the uniqueness of the minimizer.

\section{Proofs related to Section 4.1}

We report the proof of Proposition 4.1.

Proof. Using Eq. (4.1), we first note that the function $t \mapsto p_{t}$ is at least twice continuously differentiable with first derivative $p_{t}^{\prime}=\sinh \left(\frac{\epsilon t}{\sigma}\right) a_{t}$ for each $t \in[0, T]$. Therefore, we can rewrite the Lagrangian in Eq. (3.6) as

$$
\begin{aligned}
\mathcal{L}\left(t, u_{t}, u_{t}^{\prime}, p_{t}, p_{t}^{\prime}\right)= & \epsilon u_{t} \alpha_{t}-\frac{\gamma_{1}}{2 \sigma^{2}} v_{t}^{2} \alpha_{t}^{2}+\frac{1}{\sigma^{2}} \operatorname{csch}\left(\frac{\epsilon t}{\sigma}\right) v_{t} \alpha_{t} p_{t}^{\prime}-\frac{\epsilon}{\sigma^{3}} \operatorname{sech}\left(\frac{\epsilon t}{\sigma}\right) v_{t} \alpha_{t} p_{t} \\
& -\left(\frac{k}{2} u_{t}^{2}+\frac{\xi}{2}\left(\operatorname{csch}\left(\frac{\epsilon t}{\sigma}\right)\right)^{2}\left(p_{t}^{\prime}\right)^{2}\right)
\end{aligned}
$$

Our aim is to maximize $\int_{0}^{T} \mathcal{L}$ first over $u_{t}$ and then over $p_{t}$ satisfying Eqs.(4.1) and (4.2). Observe that $\mathcal{L}$ is well-defined and smooth in all variables because it is a different way of writing the smooth Lagrangian in Eq. (3.6) via Eq. (4.1). Notice that the negative Lagrangian $-\mathcal{L}$ is quadratic in $u_{t}$ and there is no $u_{t}^{\prime}$ appearing. Since $\mathcal{L}$ is strictly concave in $u_{t}$, the maximizer $u_{t}^{*}$ is unique and at least $C^{1}[0, T]$. Moreover, it is found as the critical point. Notice that $\mathcal{L}$ is quadratic $p_{t}^{\prime}$, and linear in $p_{t}$. Therefore, $-\mathcal{L}$ is convex in $\left(p_{t}, p_{t}^{\prime}\right)$. Additionally, $-\mathcal{L}$ is strictly convex in $p_{t}^{\prime}$ because

$$
-\frac{\partial^{2} \mathcal{L}}{\left(\partial p_{t}^{\prime}\right)^{2}} \geq \xi \operatorname{csch}\left(\frac{\epsilon T}{\sigma}\right)>0
$$

It can be seen that $-\mathcal{L}$ is coercive in $p_{t}^{\prime}$, as it can be bounded below by $\left(p_{t}^{\prime}\right)^{2}$. Since $\mathcal{L}$ is a smooth function in $p_{t}$ and $p_{t}^{\prime},-\mathcal{L}$ becomes a Tonelli Lagrangian. By using the celebrated theorem of Tonelli (Theorem B.2) we infer that $\int_{0}^{T}-\mathcal{L}\left(t, u_{t}, u_{t}^{\prime}, p_{t}, p_{t}^{\prime}\right) d t$ has a unique (at least $\left.C^{2}[0, T]\right)$ minimizer $p_{t}^{*}$ that satisfy the Euler-Lagrange equation. Thus, we have the following system of equations

$$
\begin{aligned}
\frac{\partial \mathcal{L}}{\partial u_{t}} & =0 \\
\frac{d}{d t}\left(\frac{\partial \mathcal{L}}{\partial p_{t}^{\prime}}\right) & =\frac{\partial \mathcal{L}}{\partial p_{t}}
\end{aligned}
$$

From Eq. (C.2), we immediately obtain that the optimal $u_{t}^{*}$ is given by Eq. (4.6). We may 
rewrite the second equation (Eq. (C.3)) as

$$
\frac{d}{d t}\left(\frac{1}{\sigma^{2}} \operatorname{csch}\left(\frac{\epsilon t}{\sigma}\right) v_{t} \alpha_{t}-\xi\left(\operatorname{csch}\left(\frac{\epsilon t}{\sigma}\right)\right)^{2}\left(p^{*}\right)_{t}^{\prime}\right)=-\frac{\epsilon}{\sigma^{3}} \operatorname{sech}\left(\frac{\epsilon t}{\sigma}\right) v_{t} \alpha_{t}
$$

Notice that $\left(p^{*}\right)_{T}$ is a free end point. Integrating from $t$ to $T$, and using the free boundary condition

$$
\left.\frac{\partial \mathcal{L}}{\partial p_{t}^{\prime}}\right|_{t=T}=0 \Longleftrightarrow \frac{1}{\sigma^{2}} \operatorname{csch}\left(\frac{\epsilon T}{\sigma}\right) \alpha_{T} v_{T}=\xi\left(\operatorname{csch}\left(\frac{\epsilon T}{\sigma}\right)\right)^{2}\left(p^{*}\right)_{T}^{\prime}
$$

we arrive at

$$
\xi\left(\operatorname{csch}\left(\frac{\epsilon t}{\sigma}\right)\right)^{2}\left(p^{*}\right)_{t}^{\prime}=\frac{\epsilon}{\sigma} \operatorname{sech}\left(\frac{\epsilon t}{\sigma}\right) \alpha_{t}-\frac{\epsilon^{2}}{\sigma^{2}} \int_{t}^{T} \tanh \left(\frac{\epsilon s}{\sigma}\right) \operatorname{sech}\left(\frac{\epsilon s}{\sigma}\right) \alpha_{s} d s
$$

after using the explicit expression for $v_{t}$ given by (2.7). From the definition of $p_{t}$ in Eqs. (4.1) and (4.2), we can express Eq. (C.6) in terms of $a_{t}$ leading to Eq. (4.5). This proves the proposition.

\section{Proofs related to Section 4.2}

Verification of Remark 4.4. First note that $\mathcal{X}$ is defined for all $t \in[0, T]$, as it is a different version of the Lagrangian (integrand) in Eq. (4.7). Furthermore, it is quadratic in $\left(\beta^{\prime}, \eta^{\prime}, \zeta^{\prime}\right)$ and smooth in all variables due to equations (4.13)-(4.14). One can easily check that $-X$ is convex in $\left(\beta_{t}, \eta_{t}, \zeta_{t}, \beta_{t}^{\prime}, \eta_{t}^{\prime}, \zeta_{t}^{\prime}\right)$. Indeed, letting $\beta_{t}=w_{1}, \eta_{t}=w_{2}, \zeta_{t}=w_{3}, \beta_{t}^{\prime}=w_{4}, \eta_{t}^{\prime}=w_{5}, \zeta_{t}^{\prime}=$ $w_{6}$ and $\mathbf{w}=\left(w_{1}, w_{2}, w_{3}, w_{4}, w_{5}, w_{6}\right)$ we have

$$
\mathcal{H}_{X} \triangleq \frac{\partial^{2} X}{\partial \mathbf{w}^{2}}=\left[\begin{array}{cccccc}
0 & 0 & 0 & 0 & 0 & 0 \\
0 & a_{22} & 0 & a_{24} & 0 & 0 \\
0 & 0 & 0 & 0 & 0 & 0 \\
0 & a_{42} & 0 & a_{44} & 0 & 0 \\
0 & 0 & 0 & 0 & 0 & 0 \\
0 & 0 & 0 & 0 & 0 & 0
\end{array}\right]
$$

where

$$
\begin{gathered}
a_{22}=-\frac{\epsilon^{4}}{\sigma^{4} \xi} \sinh ^{2}\left(\frac{\epsilon t}{\sigma}\right), \quad a_{24}=a_{42}=\frac{\epsilon^{3}}{\sigma^{3} \xi} \\
a_{44}=-\left(\frac{\epsilon^{2}}{\sigma^{2} \xi}+\epsilon^{2}\left(\gamma_{1}+\gamma_{2}\right)+\frac{\epsilon^{2}}{k} \cosh ^{2}\left(\frac{\epsilon t}{\sigma}\right)\right) \operatorname{csch}^{2}\left(\frac{\epsilon t}{\sigma}\right) .
\end{gathered}
$$

Therefore, we conclude that the eigenvalues $\mu_{i}(1 \leq i \leq 3)$ of the Hessian matrix $\mathcal{H}_{x}$ given by

$$
\mu_{1}=0, \quad \mu_{2,3}=\frac{1}{2}\left[a_{22}+a_{44} \pm \sqrt{\left(a_{44}-a_{22}\right)^{2}+4\left(a_{42}\right)^{2}}\right]
$$

are all nonpositive due to $a_{24}^{2}<a_{44} a_{22}$ for all $t \geq 0$. 
Furthermore, as a consequence of Eqs. (4.13)-(4.14), it is enough to have the coercivity and strict convexity of $-X$ in only one component of $\left(\beta^{\prime}, \eta^{\prime}, \zeta^{\prime}\right)$, as we can rewrite $X$ to make the quadratic terms $\left(\beta^{\prime}\right)^{2},\left(\eta_{t}^{\prime}\right)^{2}$ and $\left(\zeta^{\prime}\right)^{2}$ appear in $\mathcal{X}$. Therefore, it is not difficult to see that $-X$ is coercive as well as strictly convex in $\left(\beta^{\prime}, \eta^{\prime}, \zeta^{\prime}\right)$. Thus, it turns out that $-X$ is a Tonelli Lagrangian and so again by Theorem B.2 there exist unique (at least $C^{2}[0, T]$ ) maximizers $\beta, \eta, \zeta$ of $\mathcal{A}^{P}$. Hence $\beta, \eta, \zeta$ satisfy

$$
\begin{aligned}
& \max _{\alpha_{t}}\left\{\mathcal{A}\left(t, \alpha_{t}\right): a_{t}^{M E} \text { satisfies (4.5) and } u_{t}^{M E} \text { satisfies (4.6) }\right\}= \\
& \max _{\left(\beta_{t}, \eta_{t}, \zeta_{t}\right)}\left\{\mathcal{A}^{P}\left(t, \beta_{t}, \eta_{t}, \zeta_{t}\right): \beta_{t} \text { and } \eta_{t} \text { satisfy (4.13), } \eta_{t} \text { and } \zeta_{t} \text { satisfy (4.14) }\right\}
\end{aligned}
$$

The following lemma characterizes the unique maximizers $\left(\beta_{t}, \eta_{t}, \zeta_{t}\right)$ of $\mathcal{A}^{P}$.

Lemma D.3. If $\left(\beta_{t}, \eta_{t}, \zeta_{t}\right)$ maximizes $\mathcal{A}^{P}$ in Eq. (4.15) with Eq. (4.13), Eq. (4.14) and $\beta_{0}=\eta_{T}=\zeta_{0}=0$, then the following equations are satisfied:

$$
\begin{gathered}
\frac{\partial \mathcal{X}}{\partial \beta_{t}}-\frac{d}{d t}\left(\frac{\partial X}{\partial \beta_{t}^{\prime}}+\lambda_{2}(t)\right)=0 \\
\lambda_{2}(T)=-\left.\frac{\partial X}{\partial \beta_{t}^{\prime}}\right|_{t=T} \\
\frac{\partial X}{\partial \eta_{t}}-\lambda_{1}(t) \sinh ^{2}\left(\frac{\epsilon t}{\sigma}\right)=\frac{d}{d t}\left(\frac{\partial X}{\partial \eta_{t}^{\prime}}+\lambda_{2}(t) \sinh \left(\frac{\epsilon t}{\sigma}\right) \cosh \left(\frac{\epsilon t}{\sigma}\right)\right) \\
{\left[\frac{\partial X}{\partial \eta_{t}^{\prime}}+\lambda_{2}(t) \sinh \left(\frac{\epsilon t}{\sigma}\right) \cosh \left(\frac{\epsilon t}{\sigma}\right)\right]_{t=0}=0} \\
\frac{\partial X}{\partial \zeta_{t}}-\frac{d}{d t}\left[\frac{\partial X}{\partial \zeta_{t}^{\prime}}+\lambda_{1}(t)\right]=0 \\
\lambda_{1}(T)=-\left.\frac{\partial X}{\partial \zeta_{t}^{\prime}}\right|_{t=T} \\
\zeta_{t}^{\prime}=\sinh ^{2}\left(\frac{\epsilon t}{\sigma}\right) \eta_{t} \\
\beta_{t}^{\prime}=-\sinh \left(\frac{\epsilon t}{\sigma}\right) \cosh \left(\frac{\epsilon t}{\sigma}\right) \eta_{t}^{\prime}
\end{gathered}
$$

Proof. First, we define

$$
\begin{aligned}
X^{\lambda_{1}, \lambda_{2}}\left(t, \beta_{t}, \eta_{t}, \zeta_{t}, \beta_{t}^{\prime}, \zeta_{t}^{\prime}, \eta_{t}^{\prime}\right) \triangleq & x\left(t, \beta_{t}, \eta_{t}, \zeta_{t}, \beta_{t}^{\prime}, \zeta_{t}^{\prime}, \eta_{t}^{\prime}\right)+\lambda_{1}(t)\left(\zeta_{t}^{\prime}-\sinh ^{2}\left(\frac{\epsilon t}{\sigma}\right) \eta_{t}\right) \\
& +\lambda_{2}(t)\left(\beta_{t}^{\prime}+\sinh \left(\frac{\epsilon t}{\sigma}\right) \cosh \left(\frac{\epsilon t}{\sigma}\right) \eta_{t}^{\prime}\right)
\end{aligned}
$$


Consider the functional

$$
\Psi\left(\beta_{t}, \eta_{t}, \zeta_{t}\right) \triangleq \int_{0}^{T} x^{\lambda_{1}, \lambda_{2}}\left(t, \beta_{t}, \eta_{t}, \zeta_{t}, \beta_{t}^{\prime}, \eta_{t}^{\prime}, \zeta_{t}^{\prime}\right) d t
$$

Suppose that $\left(\beta_{t}, \eta_{t}, \zeta_{t}\right)$ maximizes the functional $\Psi$ defined by Eq. (D.11). Let $\tilde{\beta}_{t}, \tilde{\eta}_{t}, \tilde{\zeta}_{t}$ be smooth functions such that

$$
\tilde{\beta}_{0}=\tilde{\eta}_{T}=\tilde{\zeta}_{0}=0 .
$$

Therefore, any perturbation $\left(\beta_{t}+\delta_{1} \tilde{\beta}_{t}, \eta_{t}+\delta_{2} \tilde{\eta}_{t}, \zeta_{t}+\delta_{3} \tilde{\zeta}_{t}\right)$ of $\left(\beta_{t}, \eta_{t}, \zeta_{t}\right)$ would yield

$$
\Psi\left(\beta_{t}, \eta_{t}, \zeta\right) \geq \Psi\left(\beta_{t}+\delta_{1} \tilde{\beta}_{t}, \eta_{t}+\delta_{2} \tilde{\eta}_{t}, \zeta_{t}+\delta_{3} \tilde{\zeta}_{t}\right)
$$

for any real number $\delta_{1}, \delta_{2}$, and $\delta_{3}$. Thus, we conclude that $\left(\delta_{1}, \delta_{2}, \delta_{3}\right)=(0,0,0)$ is the critical point of the map

$$
\left(\delta_{1}, \delta_{2}, \delta_{3}\right) \mapsto \Psi\left(\beta_{t}+\delta_{1} \tilde{\beta}_{t}, \eta_{t}+\delta_{2} \tilde{\eta}_{t}, \zeta_{t}+\delta_{3} \tilde{\zeta}_{t}\right) .
$$

Therefore, we must have

$$
\left.\frac{\partial}{\partial \delta_{1}} \Psi\left(\beta_{t}+\delta_{1} \tilde{\beta}_{t}, \eta_{t}, \zeta_{t}\right)\right|_{\delta_{1}=0}=0
$$

and

$$
\left.\frac{\partial}{\partial \delta_{2}} \Psi\left(\beta_{t}, \eta_{t}+\delta_{2} \tilde{\eta}_{t}, \zeta_{t}\right)\right|_{\delta_{2}=0}=0
$$

and

$$
\left.\frac{\partial}{\partial \delta_{3}} \Psi\left(\beta_{t}, \eta_{t}, \zeta_{t}+\delta_{3} \tilde{\zeta}_{t}\right)\right|_{\delta_{3}=0}=0
$$

By (D.13) we have

$$
\int_{0}^{T}\left(\frac{\partial X}{\partial \beta_{t}} \tilde{\beta}_{t}+\frac{\partial X}{\partial \beta_{t}^{\prime}} \tilde{\beta}_{t}^{\prime}+\lambda_{2}(t) \tilde{\beta}_{t}^{\prime}\right) d t=0
$$

Application of the integration by parts yields

$$
\int_{0}^{T}\left(\frac{\partial \mathcal{X}}{\partial \beta_{t}}-\frac{d}{d t}\left[\frac{\partial \mathcal{X}}{\partial \beta_{t}^{\prime}}+\lambda_{2}(t)\right]\right) \tilde{\beta}_{t} d t+\left[\left(\frac{\partial \mathcal{X}}{\partial \beta_{t}^{\prime}}+\lambda_{2}(t)\right) \tilde{\beta}_{t}\right]_{t=0}^{t=T}=0 .
$$

This, together with the free boundary $\tilde{\beta}_{T}$, gives equations (D.2) and (D.3). Similarly, by (D.14) we have

$$
\int_{0}^{T}\left(\frac{\partial X}{\partial \eta_{t}} \tilde{\eta}_{t}+\frac{\partial X}{\partial \eta_{t}^{\prime}} \tilde{\eta}_{t}^{\prime}-\lambda_{1}(t) \sinh ^{2}\left(\frac{\epsilon t}{\sigma}\right) \tilde{\eta}_{t}+\lambda_{2}(t) \sinh \left(\frac{\epsilon t}{\sigma}\right) \cosh \left(\frac{\epsilon t}{\sigma}\right) \tilde{\eta}_{t}^{\prime}\right) d t=0 .
$$


Using the integration by parts we arrive at

$$
\begin{gathered}
\int_{0}^{T}\left(\frac{\partial X}{\partial \eta_{t}}-\lambda_{1}(t) \sinh ^{2}\left(\frac{\epsilon t}{\sigma}\right)-\frac{d}{d t}\left[\frac{\partial \mathcal{X}}{\partial \eta_{t}^{\prime}}+\lambda_{2}(t) \sinh \left(\frac{\epsilon t}{\sigma}\right) \cosh \left(\frac{\epsilon t}{\sigma}\right)\right]\right) \tilde{\eta}_{t} d t \\
+\left[\left(\frac{\partial \mathcal{X}}{\partial \eta_{t}^{\prime}}+\lambda_{2}(t) \sinh \left(\frac{\epsilon t}{\sigma}\right) \cosh \left(\frac{\epsilon t}{\sigma}\right)\right) \tilde{\eta}_{t}\right]_{t=0}^{t=T}=0
\end{gathered}
$$

from which, together with free boundary $\tilde{\eta}_{0}$, we obtain equations (D.4) and (D.5). By (D.15) we have

$$
\int_{0}^{T}\left(\frac{\partial X}{\partial \zeta_{t}} \tilde{\zeta}_{t}+\frac{\partial X}{\partial \zeta_{t}^{\prime}} \tilde{\zeta}_{t}^{\prime}+\lambda_{1}(t) \tilde{\zeta}_{t}^{\prime}\right) d t=0
$$

Application of the integration by parts yields

$$
\int_{0}^{T}\left(\frac{\partial X}{\partial \zeta_{t}}-\frac{d}{d t}\left[\frac{\partial X}{\partial \zeta_{t}^{\prime}}+\lambda_{1}(t)\right]\right) \tilde{\zeta}_{t} d t+\left[\left(\frac{\partial X}{\partial \zeta_{t}^{\prime}}+\lambda_{1}(t)\right) \tilde{\zeta}_{t}\right]_{t=0}^{t=T}=0
$$

With the aid of free boundary $\tilde{\zeta}_{T}$, we obtain equations (D.6) and (D.7).

We report the proof of Theorem 4.1,

Proof. Here, we use Lemma D.3, and compute equations (D.2)-(D.7), using the relations defined by Eq. (D.8) and (D.9). Note that using $v_{t}=\epsilon \sigma \tanh \left(\frac{\epsilon t}{\sigma}\right)$ we get

$$
\frac{\partial X}{\partial \beta_{t}}=-\frac{\epsilon^{2} v_{t}}{\sigma^{4} \xi} \operatorname{sech}\left(\frac{\epsilon t}{\sigma}\right)=\frac{\epsilon^{2}}{\sigma^{2} \xi} \frac{d}{d t}\left[\operatorname{sech}\left(\frac{\epsilon t}{\sigma}\right)\right]
$$

Also,

$$
\begin{aligned}
\frac{\partial X}{\partial \beta_{t}^{\prime}}= & \frac{\epsilon^{2}}{k} \operatorname{csch}\left(\frac{\epsilon t}{\sigma}\right) \operatorname{coth}\left(\frac{\epsilon t}{\sigma}\right)-\epsilon^{2}\left(\gamma_{1}+\gamma_{2}\right)\left(\operatorname{csch}\left(\frac{\epsilon t}{\sigma}\right)\right)^{2} \beta_{t}^{\prime}+\epsilon^{2}\left(\gamma_{2}+\frac{1}{\sigma^{2} \xi}\right) \operatorname{sech}\left(\frac{\epsilon t}{\sigma}\right) \\
& -\epsilon^{2} \operatorname{csch}^{2}\left(\frac{\epsilon t}{\sigma}\right)\left(\frac{1}{\sigma^{2} \xi}+\frac{1}{k} \operatorname{coth}^{2}\left(\frac{\epsilon t}{\sigma}\right)\right) \beta_{t}^{\prime}+\frac{\epsilon^{3}}{\sigma^{3} \xi} \eta_{t}
\end{aligned}
$$

Therefore, Eq. (D.2) can be written as

$$
\frac{d}{d t}\left[\frac{\epsilon^{2}}{\sigma^{2} \xi} \operatorname{sech}\left(\frac{\epsilon t}{\sigma}\right)-\frac{\partial X}{\partial \beta_{t}^{\prime}}-\lambda_{2}(t)\right]=0
$$

Integrating Eq. (D.18) from $t$ to $T$ and using equations (D.17) and (D.9) together with the boundary condition given by Eq. (D.3), we get

$$
\begin{aligned}
\lambda_{2}(t)= & \left(-\frac{\epsilon^{2}}{k} \operatorname{coth}^{2}\left(\frac{\epsilon t}{\sigma}\right)-\epsilon^{2}\left(\gamma_{1}+\gamma_{2}+\frac{1}{\sigma^{2} \xi}\right)\right) \operatorname{coth}\left(\frac{\epsilon t}{\sigma}\right) \eta_{t}^{\prime}-\frac{\epsilon^{3}}{\sigma^{3} \xi} \eta_{t} \\
& -\frac{\epsilon^{2}}{\sigma^{2} \xi} \operatorname{sech}\left(\frac{\epsilon T}{\sigma}\right)-\gamma_{2} \epsilon^{2} \operatorname{sech}\left(\frac{\epsilon t}{\sigma}\right)-\frac{\epsilon^{2}}{k} \operatorname{csch}\left(\frac{\epsilon t}{\sigma}\right) \operatorname{coth}\left(\frac{\epsilon t}{\sigma}\right)
\end{aligned}
$$


Also, using (D.9), we have

$$
\frac{\partial X}{\partial \eta_{t}}=-\frac{\epsilon^{3}}{\sigma^{3} \xi} \sinh \left(\frac{\epsilon t}{\sigma}\right) \cosh \left(\frac{\epsilon t}{\sigma}\right) \eta_{t}^{\prime}-\frac{\epsilon^{4}}{\sigma^{4} \xi} \sinh ^{2}\left(\frac{\epsilon t}{\sigma}\right) \eta_{t}-\frac{\epsilon^{3}}{\sigma^{3} \xi} \sinh ^{2}\left(\frac{\epsilon t}{\sigma}\right) \operatorname{sech}\left(\frac{\epsilon t}{\sigma}\right)
$$

Since

$$
\frac{\partial x}{\partial \eta_{t}^{\prime}}=0
$$

Eq. (D.5) becomes

$$
\left[\lambda_{2}(t) \sinh \left(\frac{\epsilon t}{\sigma}\right) \cosh \left(\frac{\epsilon t}{\sigma}\right)\right]_{t=0}=0
$$

Using the definition of $\eta_{t}$ in Eq. (4.10) we have that

$$
\eta_{t}^{\prime}=-\tanh \left(\frac{\epsilon t}{\sigma}\right) \operatorname{sech}\left(\frac{\epsilon t}{\sigma}\right) \alpha_{t}
$$

which together with (D.22) produce the boundary condition $\alpha_{0}=1$. As a result, due to Eq. (D.23), we have the boundary condition $\eta_{0}^{\prime}=0$ in Eq. (4.17).

To use Eq. (D.4) we need to compute (D.6) and (D.7). Note that

$$
\frac{\partial X}{\partial \zeta_{t}}=\frac{\epsilon^{3} v_{t}}{\sigma^{5} \xi} \operatorname{sech}\left(\frac{\epsilon t}{\sigma}\right)=-\frac{\epsilon^{3}}{\sigma^{3} \xi} \frac{d}{d t}\left[\operatorname{sech}\left(\frac{\epsilon t}{\sigma}\right)\right] .
$$

Since

$$
\frac{\partial x}{\partial \zeta_{t}^{\prime}}=0
$$

Eq. (D.7) becomes

$$
\lambda_{1}(T)=0 .
$$

Using equations (D.24) and (D.6) we obtain

$$
\frac{d}{d t}\left[-\frac{\epsilon^{3}}{\sigma^{3} \xi} \operatorname{sech}\left(\frac{\epsilon t}{\sigma}\right)-\lambda_{1}(t)\right]=0
$$

Integrating (D.27) from $t$ to $T$ and using Eq. (D.26) we get

$$
\lambda_{1}(t)=\frac{\epsilon^{3}}{\sigma^{3} \xi}\left[\operatorname{sech}\left(\frac{\epsilon T}{\sigma}\right)-\operatorname{sech}\left(\frac{\epsilon t}{\sigma}\right)\right]
$$

Now substituting equations (D.28) and (D.19) into (D.4) we obtain

$$
\begin{aligned}
\frac{\partial X}{\partial \eta_{t}}-\lambda_{1}(t) \sinh ^{2}\left(\frac{\epsilon t}{\sigma}\right)= & -\frac{\epsilon^{3}}{\sigma^{3} \xi} \sinh \left(\frac{\epsilon t}{\sigma}\right) \cosh \left(\frac{\epsilon t}{\sigma}\right) \eta_{t}^{\prime}-\frac{\epsilon^{4}}{\sigma^{4} \xi} \sinh ^{2}\left(\frac{\epsilon t}{\sigma}\right) \eta_{t} \\
& -\frac{\epsilon^{3}}{\sigma^{3} \xi} \sinh ^{2}\left(\frac{\epsilon t}{\sigma}\right) \operatorname{sech}\left(\frac{\epsilon T}{\sigma}\right)
\end{aligned}
$$




$$
\begin{aligned}
\frac{d}{d t}\left(\frac{\partial X}{\partial \eta_{t}^{\prime}}+\lambda_{2}(t) \sinh \left(\frac{\epsilon t}{\sigma}\right) \cosh \left(\frac{\epsilon t}{\sigma}\right)\right)= & -\frac{\epsilon^{3}}{\sigma^{3} \xi} \operatorname{sech}\left(\frac{\epsilon T}{\sigma}\right)\left[\cosh ^{2}\left(\frac{\epsilon t}{\sigma}\right)+\sinh ^{2}\left(\frac{\epsilon t}{\sigma}\right)\right] \\
& +\frac{\epsilon^{3}}{\sigma k} \cosh \left(\frac{\epsilon t}{\sigma}\right)\left[\operatorname{csch}^{2}\left(\frac{\epsilon t}{\sigma}\right)-1-k \gamma_{2}\right] \\
& -\frac{\epsilon^{4}}{\sigma^{4} \xi}\left[\cosh ^{2}\left(\frac{\epsilon t}{\sigma}\right)+\sinh ^{2}\left(\frac{\epsilon t}{\sigma}\right)\right] \eta_{t} \\
& -\frac{\epsilon^{3}}{\sigma^{3} \xi} \sinh \left(\frac{\epsilon t}{\sigma}\right) \cosh \left(\frac{\epsilon t}{\sigma}\right) \eta_{t}^{\prime} \\
& -\frac{2 \epsilon^{3}}{\sigma}\left(\gamma_{1}+\gamma_{2}+\frac{1}{\sigma^{2} \xi}\right) \sinh \left(\frac{\epsilon t}{\sigma}\right) \cosh \left(\frac{\epsilon t}{\sigma}\right) \eta_{t}^{\prime} \\
& -\frac{2 \epsilon^{3}}{\sigma k} \operatorname{coth}\left(\frac{\epsilon t}{\sigma}\right)\left(\cosh ^{2}\left(\frac{\epsilon t}{\sigma}\right)-\operatorname{coth}^{2}\left(\frac{\epsilon t}{\sigma}\right)\right) \eta_{t}^{\prime} \\
& -\epsilon^{2}\left(\gamma_{1}+\gamma_{2}+\frac{1}{\sigma^{2} \xi}\right) \cosh ^{2}\left(\frac{\epsilon t}{\sigma}\right) \eta_{t}^{\prime \prime} \\
& -\frac{\epsilon^{2}}{k} \operatorname{coth}^{2}\left(\frac{\epsilon t}{\sigma}\right) \cosh ^{2}\left(\frac{\epsilon t}{\sigma}\right) \eta_{t}^{\prime \prime}
\end{aligned}
$$

Substitution of equations (D.29) and (D.30) into Equation (D.4) results in the differential equation given by (4.17), which completes the proof.

\section{E Proofs related to Section 4.3}

We report the proof of Proposition 4.2

Proof. It is enough to prove that the pay-per-performance sensitivities are the same, as the same relation between $u_{t}$ and $\alpha_{t}$ persists in both contracting models (compare Eq. (4.6) and Eq. (4.25)). We first compute the limiting behavior as $\xi \rightarrow \infty$ of the ODE given in (4.17):

$$
\begin{gathered}
\eta_{t}^{\prime}=-\tanh \left(\frac{\epsilon t}{\sigma}\right) \operatorname{sech}\left(\frac{\epsilon t}{\sigma}\right) \alpha_{t} \\
\eta_{t}^{\prime \prime}=-\tanh \left(\frac{\epsilon t}{\sigma}\right) \operatorname{sech}\left(\frac{\epsilon t}{\sigma}\right) \alpha_{t}^{\prime}-\frac{\epsilon}{\sigma} \operatorname{sech}^{3}\left(\frac{\epsilon t}{\sigma}\right) \alpha_{t}+\frac{\epsilon}{\sigma} \tanh ^{2}\left(\frac{\epsilon t}{\sigma}\right) \operatorname{sech}\left(\frac{\epsilon t}{\sigma}\right) \alpha_{t}(\mathrm{E} \\
A(t)^{(\xi \rightarrow \infty)} \eta_{t}^{\prime \prime}=\frac{\epsilon^{3}}{\sigma}\left(\gamma_{1}+\gamma_{2}+\frac{1}{k} \operatorname{coth}\left(\frac{\epsilon t}{\sigma}\right)\right)\left(\tanh \left(\frac{\epsilon t}{\sigma}\right) \sinh \left(\frac{\epsilon t}{\sigma}\right)-\operatorname{sech}\left(\frac{\epsilon t}{\sigma}\right)\right) \alpha_{t} \\
-\epsilon^{2}\left(\gamma_{1}+\gamma_{2}+\frac{1}{k} \operatorname{coth}\left(\frac{\epsilon t}{\sigma}\right)\right) \sinh \left(\frac{\epsilon t}{\sigma}\right) \alpha_{t}^{\prime}
\end{gathered}
$$




$$
\begin{aligned}
& B(t)^{(\xi \rightarrow \infty)} \eta_{t}^{\prime}=-\frac{2 \epsilon^{3}}{\sigma}\left(\gamma_{1}+\gamma_{2}\right) \sinh \left(\frac{\epsilon t}{\sigma}\right) \tanh \left(\frac{\epsilon t}{\sigma}\right) \alpha_{t} \\
&-\frac{2 \epsilon^{3}}{\sigma k} \operatorname{sech}\left(\frac{\epsilon t}{\sigma}\right)\left(\cosh ^{2}\left(\frac{\epsilon t}{\sigma}\right)-\operatorname{coth}^{2}\left(\frac{\epsilon t}{\sigma}\right)\right) \alpha_{t} \\
& C(t)^{(\xi \rightarrow \infty)} \eta_{t}=0 \\
& D(t)^{(\xi \rightarrow \infty)=}=\frac{\epsilon^{3}}{\sigma} \cosh \left(\frac{\epsilon t}{\sigma}\right)\left(\gamma_{2}+\frac{1}{k}\left(1-\operatorname{csch}^{2}\left(\frac{\epsilon t}{\sigma}\right)\right)\right)
\end{aligned}
$$

Using equations (E.3) $-(\underline{\text { E.6 }})$ in (4.17) and dividing everything by $\cosh \left(\frac{\epsilon t}{\sigma}\right)$ and multiplying by $\sigma$, we arrive at

$$
\begin{aligned}
0= & \epsilon^{3}\left(\gamma_{1}+\gamma_{2}+\frac{1}{k} \operatorname{coth}\left(\frac{\epsilon t}{\sigma}\right)\right)\left(\tanh ^{2}\left(\frac{\epsilon t}{\sigma}\right)-\operatorname{sech}^{2}\left(\frac{\epsilon t}{\sigma}\right)\right) \alpha_{t} \\
& -\epsilon^{2} \sigma\left(\gamma_{1}+\gamma_{2}+\frac{1}{k} \operatorname{coth}\left(\frac{\epsilon t}{\sigma}\right)\right) \tanh \left(\frac{\epsilon t}{\sigma}\right) \alpha_{t}^{\prime} \\
& -2 \epsilon^{3}\left(\gamma_{1}+\gamma_{2}\right) \tanh ^{2}\left(\frac{\epsilon t}{\sigma}\right) \alpha_{t} \\
& -\frac{2 \epsilon^{3}}{k} \operatorname{sech}^{2}\left(\frac{\epsilon t}{\sigma}\right)\left(\cosh ^{2}\left(\frac{\epsilon t}{\sigma}\right)-\operatorname{coth}^{2}\left(\frac{\epsilon t}{\sigma}\right)\right) \alpha_{t} \\
& +\epsilon^{3}\left(\gamma_{2}+\frac{1}{k}\left(1-\operatorname{csch}^{2}\left(\frac{\epsilon t}{\sigma}\right)\right)\right)
\end{aligned}
$$

Observe that

$$
\lim _{\sigma \rightarrow 0} \tanh \left(\frac{\epsilon t}{\sigma}\right)=\lim _{\sigma \rightarrow 0} \operatorname{coth}\left(\frac{\epsilon t}{\sigma}\right)=1, \quad \lim _{\sigma \rightarrow 0} \operatorname{sech}\left(\frac{\epsilon t}{\sigma}\right)=\lim _{\sigma \rightarrow 0} \operatorname{csch}\left(\frac{\epsilon t}{\sigma}\right)=0 .
$$

Taking the limit as $\sigma \rightarrow 0$ in Equation (E.7), we get

$$
-\epsilon^{3}\left(\gamma_{1}+\gamma_{2}+\frac{1}{k}\right) \alpha_{t}+\epsilon^{3}\left(\gamma_{2}+\frac{1}{k}\right)=0
$$

which together with Eq. (4.25) gives Eq. (4.29).

We report the proof of Proposition 4.3

Proof. We start computing the limiting behavior of the ODE given by (4.17) as $k \rightarrow \infty$. We obtain

$$
\begin{gathered}
A(t)^{(k \rightarrow \infty)}=\epsilon^{2}\left(\gamma_{1}+\gamma_{2}+\frac{1}{\sigma^{2} \xi}\right) \cosh \left(\frac{\epsilon t}{\sigma}\right)^{2} \\
B(t)^{(k \rightarrow \infty)}=\frac{2 \epsilon^{3}}{\sigma}\left(\gamma_{1}+\gamma_{2}+\frac{1}{\sigma^{2} \xi}\right) \sinh \left(\frac{\epsilon t}{\sigma}\right) \cosh \left(\frac{\epsilon t}{\sigma}\right)
\end{gathered}
$$




$$
\begin{gathered}
C(t)^{(k \rightarrow \infty)}=\frac{\epsilon^{4}}{\sigma^{4} \xi} \cosh \left(\frac{\epsilon t}{\sigma}\right)^{2} \\
D(t)^{(k \rightarrow \infty)}=\frac{\epsilon^{3}}{\sigma^{3} \xi} \cosh \left(\frac{\epsilon t}{\sigma}\right)^{2} \operatorname{sech}\left(\frac{\epsilon T}{\sigma}\right)+\frac{\epsilon^{3} \gamma_{2}}{\sigma} \cosh \left(\frac{\epsilon t}{\sigma}\right)
\end{gathered}
$$

Plugging equations (E.9) $-(\underline{E .12})$ into (4.17) and using the boundary conditions $\eta_{T}=0$ and $\left.\eta_{t}^{\prime}\right|_{t=0}=0$, we obtain that the second order ODE becomes nonsingular and admits the following unique solution:

$$
\eta_{t}=\varrho\left[\left(\gamma_{1} \cosh (\omega t)+\gamma_{2} \cosh (\omega T)\right) \operatorname{sech}\left(\frac{\epsilon t}{\sigma}\right)-\left(\gamma_{1}+\gamma_{2}\right) \cosh (\omega T) \operatorname{sech}\left(\frac{\epsilon T}{\sigma}\right)\right]
$$

where $\omega$ is as in Eq. (4.28) and

$$
\rho=\frac{\epsilon \sigma \operatorname{sech}(\omega T)}{\left(\gamma_{1}+\gamma_{2}\right)\left(\epsilon^{2}-\omega^{2} \sigma^{2}\right)\left(1+\left(\gamma_{1}+\gamma_{2}\right) \xi \sigma^{2}\right)}
$$

From Eq. (4.22), we get $\alpha_{t}^{M E}=\alpha_{t}^{M}$ after differentiating (E.13), and multiplying it by the expression $-\operatorname{coth}\left(\frac{\epsilon t}{\sigma}\right) \cosh \left(\frac{\epsilon t}{\sigma}\right)$. Using the relation between $\alpha_{t}^{M E}$ and $a_{t}^{M E}$ given by Eq. (4.5), we get that $a_{t}^{M E}=a_{t}^{M}$, thereby proving the proposition.

\section{F Proofs related to limiting cases}

\section{F.0.4 Limiting cases of pay-per-performance sensitivity}

Proof of Eq. (4.31). Multiplying the ODE (and the boundary conditions) by $\xi$, and taking the limit as $\xi \rightarrow 0$, we obtain that the ordinary differential equation reduces to

$$
A^{\xi \rightarrow 0}(t) \eta_{t}^{\prime \prime}+B^{\xi \rightarrow 0}(t) \eta_{t}^{\prime}+C^{\xi \rightarrow 0}(t) \eta_{t}+D^{\xi \rightarrow 0}(t)=0
$$

with boundary conditions given by $\eta_{T}=0$ and $\eta_{0}^{\prime}=0$. Here

$$
\begin{aligned}
A^{\xi \rightarrow 0}(t) & =\frac{\epsilon^{2}}{\sigma^{2}} \cosh ^{2}\left(\frac{\epsilon t}{\sigma}\right) \\
B^{\xi \rightarrow 0}(t) & =2 \frac{\epsilon^{3}}{\sigma^{3}} \sinh \left(\frac{\epsilon t}{\sigma}\right) \cosh \left(\frac{\epsilon t}{\sigma}\right) \\
C^{\xi \rightarrow 0}(t) & =\frac{\epsilon^{4}}{\sigma^{4}} \cosh ^{2}\left(\frac{\epsilon t}{\sigma}\right) \\
D^{\xi \rightarrow 0}(t) & =\frac{\epsilon^{3}}{\sigma^{3}} \cosh ^{2}\left(\frac{\epsilon t}{\sigma}\right) \operatorname{sech}\left(\frac{\epsilon T}{\sigma}\right)
\end{aligned}
$$

It can be checked that the solution of the ODE in Eq. (F.1) is given by

$$
\eta_{t}^{\xi \rightarrow 0}=\frac{\sigma}{\epsilon}\left(\operatorname{sech}\left(\frac{\epsilon t}{\sigma}\right)-\operatorname{sech}\left(\frac{\epsilon T}{\sigma}\right)\right)
$$


and, using Eq. (4.22), we obtain $\lim _{\xi \rightarrow 0} \alpha_{t}^{M E}=1$. Using relations (4.24), and (4.23), we then obtain Eq. (4.31).

\section{F.0.5 Limiting cases $k \rightarrow 0$}

Multiplying the ODE (and the boundary conditions) by $k$, and taking the limit as $k \rightarrow 0$, we obtain that the ordinary differential equation reduces to

$$
A^{k \rightarrow 0}(t) \eta_{t}^{\prime \prime}+B^{k \rightarrow 0}(t) \eta_{t}^{\prime}+C^{k \rightarrow 0}(t) \eta_{t}+D^{k \rightarrow 0}(t)=0
$$

with boundary conditions given by $\eta_{T}=0$ and $\eta_{0}^{\prime}=0$. Here

$$
\begin{aligned}
A^{k \rightarrow 0}(t) & =\epsilon^{2} \cosh \left(\frac{\epsilon t}{\sigma}\right)^{4} \sinh \left(\frac{\epsilon t}{\sigma}\right) \\
B^{k \rightarrow 0}(t) & =\frac{2 \epsilon^{3}}{\sigma} \cosh \left(\frac{\epsilon t}{\sigma}\right)^{3} \sinh \left(\frac{\epsilon t}{\sigma}\right) \sinh \left(\frac{\epsilon t}{\sigma}\right)-\frac{2 \epsilon^{3}}{\sigma} \cosh \left(\frac{\epsilon t}{\sigma}\right)^{3} \\
C^{k \rightarrow 0}(t) & =0 \\
D^{k \rightarrow 0}(t) & =\frac{\epsilon^{3}}{\sigma} \cosh \left(\frac{\epsilon t}{\sigma}\right) \sinh \left(\frac{\epsilon t}{\sigma}\right)\left(\sinh \left(\frac{\epsilon t}{\sigma}\right)^{2}-1\right)
\end{aligned}
$$

It can be easily checked that the solution $\eta_{t}^{k \rightarrow 0}$ is given by

$$
\eta_{t}^{k \rightarrow 0}=\frac{\sigma\left(\operatorname{sech}\left(\frac{\epsilon t}{\sigma}\right)-\operatorname{sech}\left(\frac{\epsilon T}{\sigma}\right)\right)}{\epsilon}
$$

and, using Eq. (4.22), we obtain $\lim _{k \rightarrow 0} \alpha_{t}^{M E}=1$. Using relations (4.24), and (4.23), we then obtain Eq. (4.32).

\section{References}

Auzinger, W., Kneisl, G., Koch, O., and Weinmuller, E: SBVP 1.0 - A MATLAB Solver for Singular Boundary Value Problems, Technical Report, Vienna University of Technology, 2011.

Balsam, S., Bartov, E., and Marquardt, C. (2002): Accrual management, investor sophistication, and equity valuation: Evidence from 10-Q filings, Journal of Accounting Research, 40, 4, 987-1012.

Biais, B, Mariotti, T., Plantin , G. and Rochet, J-C (2007): Dynamic Security Design: Convergence to Continuous Time and Asset Pricing Implications. Review of Economic Studies, 74(2), 345-390.

Cadenillas, A., Cvitanić, J., and Zapatero, F. (2007): Optimal Risk-Sharing with Effort and Project Choice. Journal of Economic Theory 133 403-440. 
Capponi, A., and Cvitanić, J. (2009): Credit Risk Modeling with misreporting and Incomplete Information. International J. of Theoretical and Applied Finance 12 81-112.

Capponi, A., Cvitanić., J., Yolcu, T. (2011): Contracting with Hidden States and misreporting. http: //papers. ssrn. com/sol3/papers. cfm? abstract_id=1913280.

Clarke, F. H. (1989): Methods of dynamics and nonsmooth optimization Capital city press, Montpelier, Vermont 57 pages. 30-40.

Cvitanić, J., Zhang, J. (2007): Optimal Compensation with Adverse Selection and Dynamic Actions. Mathematics and Financial Economics $121-55$.

Cvitanić, J., Wan, X., and Zhang, J. (2009): Optimal Compensation with Hidden Action and Lump-Sum Payment in a Continuous-Time Model. Applied Mathematics and Optimization 59 99-146.

DeMarzo, P.M., and Sannikov, Y. (2006): Optimal Security Design and Dynamic Capital Structure in a Continuous-Time Agency Model. Journal of Finance 61 2681-2724.

Duffie, D., and Lando, D. (2001): Term structure of credit spreads with incomplete accounting information. Econometrica 63 633-664.

Ferrarin, G. and Giudici, P. (2005): Financial scandals and the role of private enforcement: the Parmalat case. ECGI Law Working Paper 40.

Gibbons, R. (2010): Lecture Note 1: Agency Theory. Prepint available at http://web.mit.edu/rgibbons/www/903LN1S10.pdf

Jensen, M. (2005): Agency Costs of Overvalued Equity. Financial Management 34, No. 1.

Holmstrom, B., and Milgrom, P. (1987): Aggregation and Linearity in the Provision of Intertemporal Incentives. Econometrica 55(2) 303-328.

Liptser, R.S., Shiryaev, A.N. (2000): Statistics of Random Processes II. Applications, Springer-Verlag, New York.

Müller, H. (1998): The First-Best Sharing Rule in the Continuous-Time Principal-Agent Problem with Exponential Utility. Journal of Economic Theory 79 276-280.

Ou-Yang, H. (2005): An equilibrium model of asset pricing and moral hazard. Review of Financial Studies 18 1253-1303.

Schättler, H., and Sung, J. (1993): The First-Order Approach to Continuous-Time PrincipalAgent Problem with Exponential Utility. Journal of Economic Theory 61 331-371.

Schättler, H., Sung, J. (1997): On Optimal Sharing Rules in Discrete and Continuous-Times Principal-Agent Problems with Exponential Utility. Journal of Economic Dynamics and Control 21 551-574. 
Sung, J. (1995): Linearity with Project Selection and Controllable Diffusion Rate in Continuous-Time Principal-Agent Problems. Rand Journal of Economics 26 720-743.

Sung, J. (1997): Corporate Insurance and Managerial Incentives. Journal of Economic Theory $74297-332$.

Tonelli, L. (1915): Sur une mithode directe du calcd des variations. Rend. Circ. Mat. Palermo 39 